\title{
On Homogenization and Scaling Limit of Some Gradient Perturbations of a Massless Free Field
}

\author{
Ali Naddaf ${ }^{1, \star}$, Thomas Spencer ${ }^{2}$ \\ 1 Courant Institute of Mathematical Sciences, 251 Mercer Street, New York, NY 10012, USA \\ 2 School of Mathematics, Institute for Advanced Study, Princeton, NJ 08540, USA
}

Received: 18 December 1995 / Accepted: 11 March 1996

\begin{abstract}
We study the continuum scaling limit of some statistical mechanical models defined by convex Hamiltonians which are gradient perturbations of a massless free field. By proving a central limit theorem for these models, we show that their long distance behavior is identical to a new (homogenized) continuum massless free field. We shall also obtain some new bounds on the 2-point correlation functions of these models.
\end{abstract}

\section{Introduction and Statement of the Main Results}

In this article, we study the long distance behavior of (lattice) statistical mechanical models defined by convex Hamiltonians $H(\varphi)$ which are gradient perturbations of a massless free Gaussian. Under certain assumptions (see ( $\mathrm{H}-1)$ and $(\mathrm{H}-2)$ below), we shall prove a central limit theorem for these models and show that their behavior at long distances is governed by suitable continuum massless Gaussians. The main idea is that these statistical mechanical models can be expressed (following Helffer and Sjöstrand $[8,12])$ in terms of an infinite dimensional elliptic PDE. We develop a suitable extension of homogenization for this elliptic equation which will then yield the desired central limit theorem. Along the way, we will obtain new estimates which are pointwise versions of the Brascamp-Lieb inequalities [1]. When the Hamiltonian is a "small" perturbation of a massless free field, using the renormalization group analysis and multi-scale expansions, these models have been studied in $[4,10]$ and more recently in [2]. Their methods, when applicable, give more detailed information than our methods. However, our methods do not require the Hamiltonian to be a "small" perturbation of a massless free field.

In this introduction, we first define the model and then we state the main results. Then the notations for the rest of paper are introduced. A brief sketch of the proofs and organization of the rest of paper conclude the introduction.

1.1. The model. At each point $x$ of the lattice $\mathbb{Z}^{d}$, there is a real random variable $\varphi(x)$ and we consider the following Hamiltonian $H_{m}^{\Lambda}, \Lambda=\Lambda(L) \subset \mathbb{Z}^{d}$ a cube of side

\footnotetext{
^ Present address: Department of Mathematics, The University of British Columbia, Vancouver, B.C. V6T 1Z2, Canada
} 
$L$ centered at the origin:

$$
H_{m}^{\Lambda}(\varphi) \stackrel{\text { def. }}{=} \sum_{\substack{x, y \in \Lambda \\\|x-y\|=1}} V(\varphi(x)-\varphi(y))+\frac{m^{2}}{2} \sum_{x \in \Lambda} \varphi(x)^{2}
$$

and $V \in C^{2}(\mathbb{R})$ is an even function. $m$ in the Hamiltonian will be referred to as the mass and the second term is called the mass term. The superscript $\Lambda$ indicates Dirichlet or periodic boundary conditions. In this article, the particular choice of boundary conditions will become unimportant as $\Lambda \uparrow \infty$. We denote by $d \mu_{m}^{\Lambda}(\varphi)$ the induced probability measure and for any allowed functional $F(\varphi)$, we use either of the following symbols to represent its expectation:

$$
\langle F\rangle_{m}^{\Lambda} \equiv \int F(\varphi) d \mu_{m}^{\Lambda}(\varphi) \stackrel{\text { def. }}{=} \frac{1}{\mathscr{E}_{m}^{\Lambda}} \int F(\varphi) \exp \left\{-H_{m}^{\Lambda}(\varphi)\right\} \mathscr{D}_{\Lambda} \varphi
$$

where

$$
\mathscr{Z}_{m}^{\Lambda}=\int_{\mathbb{R}^{|\Lambda| d}} \exp \left\{-H_{m}^{\Lambda}(\varphi)\right\} \mathscr{D}_{\Lambda} \varphi
$$

is the partition function (in volume $\Lambda$ ) and

$$
\mathscr{D}_{\Lambda \varphi} \stackrel{\text { def. }}{=} \prod_{x \in \Lambda} d \varphi(x)
$$

Note that when $V(z)=\frac{1}{2} z^{2}, H_{m}$ is a free field Hamiltonian of mass $m$.

We restrict the class of Hamiltonians by imposing the following lower bound on their Hessians as quadratic forms $(m \geq 0)$ :

$$
\text { Hess } H_{m}^{\Lambda} \stackrel{\text { def. }}{=}\left[\frac{\partial^{2} H_{m}^{\Lambda}}{\partial \varphi(x) \partial \varphi(y)}\right] \geq\left(-\delta \Delta+m^{2}\right),
$$

for some $\delta>0$, independent of $\Lambda$. Let us denote the variance of a functional $g$ by $\operatorname{Var} g$ :

$$
\operatorname{Var} g \stackrel{\text { def. }}{=}\left\langle\left(g-\langle g\rangle_{m}^{\Lambda}\right)^{2}\right\rangle_{m}^{\Lambda}
$$

and let

$$
\varphi(f) \stackrel{\text { def. }}{=} \sum_{x} \varphi(x) f(x) .
$$

We shall frequently use the following inequalities.

\section{Theorem (Brascamp-Lieb Inequalities).}

(a) For any $C^{1}$ functional $g(\varphi)$,

$$
\operatorname{Var} g(\varphi) \leq \sum_{x, y}\left\langle\frac{\partial g(\varphi)}{\partial \varphi(x)}\left(\operatorname{Hess} H_{m}^{\Lambda}\right)^{-1}(x, y) \frac{\partial g(\varphi)}{\partial \varphi(y)}\right\rangle_{m}^{\Lambda}
$$

(b)

$$
\left\langle\mathrm{e}^{t \varphi(f)}\right\rangle_{m}^{\Lambda} \leq \exp \left\{\frac{t^{2}}{2}\left(f,(-\delta \Delta+m)^{-1} f\right)\right\}
$$


For a proof, see [1] (see also Remark 6 below).

We can define an infinite volume massless (i.e. $m=0$ ) probability measure $\mu(\varphi)$ through the following procedure ${ }^{1}$ :

$$
\left\langle\mathrm{e}^{c \varphi(f)}\right\rangle \equiv \int \mathrm{e}^{c \varphi(f)} d \mu(\varphi) \stackrel{\text { def. }}{=} \lim _{m \downarrow 0}\left\langle\mathrm{e}^{c \varphi(f)}\right\rangle_{m} \stackrel{\text { def. }}{=} \lim _{m \downarrow 0} \lim _{\Lambda \uparrow \infty}\left\langle\mathrm{e}^{c \varphi(f)}\right\rangle_{m}^{\Lambda} .
$$

Note that by part (b) of Brascamp-Lieb inequalities (hereafter B-L), the right-hand side of this equation remains bounded as $m \downarrow 0$ whenever $f$ is compactly supported (and has average zero in $d=1,2$ ). The uniqueness of limit is established in [13]. Note that in $d=1,2$, the condition that $f$ has to have zero average means that the class of allowed functionals is restricted to those which only depend on the difference of fields at different points of space.

One can also use B-L inequalities to prove the ergodicity of the infinite volume probability measure constructed above. This holds independent of the boundary conditions.

To probe the long distance behavior of massless models, we introduce the slowly varying scaled field

$$
\varphi\left(f^{\varepsilon}\right) \equiv \sum_{x} \varphi(x) f^{\varepsilon}(x) \stackrel{\text { def. }}{=} \varepsilon^{d / 2+1} \sum_{x} \varphi(x) f(\varepsilon x), \quad f \in C_{0}^{\infty}\left(\mathbb{R}^{d}\right), \quad \varepsilon>0 .
$$

If $f$ is an approximation to the characteristic function of a unit box, then $\varphi\left(f^{\varepsilon}\right)$ will be the sum of $L^{d}(L \sim 1 / \varepsilon)$ random variables $\varphi(x)$ divided by the scaling factor $L^{d / 2+1}$. If $\{\varphi(x)\}$ is a family of independent (or even weakly dependent) random variables, the correct scaling for having a central limit theorem (CLT) will be $L^{-d / 2}$ but in the massless models, the field variables are strongly dependent and we require a stronger scaling, namely the factor $L^{-1-d / 2}$, to obtain a non-trivial CLT. However, the family of random variables $\{\nabla \varphi(x)\}$ only needs the standard scaling $L^{-d / 2}$. Although in $d=1$ these gradient random variables are independent, this is no longer true in $d>1$ where even at large separations, gradient fields are correlated and the correlation functions are not absolutely summable but are bounded as quadratic forms and allows the standard scaling.

Define $\nabla_{i} f(x) \stackrel{\text { def. }}{=} f\left(x+e_{i}\right)-f(x)$ and let $\nabla_{i}^{*}$ denote its adjoint with respect to the standard inner product of $\ell^{2}\left(\mathbb{Z}^{d}\right)$ (see Sect. 1.2 for more details). To cover all dimensions in a uniform way, and according to the above argument, we choose to study the gradient of fields and for $f \in C_{0}^{\infty}\left(\mathbb{R}^{d}\right)$ and an arbitrary integer $\ell, 1 \leq \ell \leq d$, we introduce

$$
\nabla_{\ell} \varphi\left(f_{\varepsilon}\right) \equiv \varphi\left(\nabla_{\ell}^{*} f_{\varepsilon}\right) \equiv \sum_{x} \varphi(x) \nabla_{\ell}^{*} f_{\varepsilon}(x), \quad f_{\varepsilon}(x) \stackrel{\text { def. }}{=} \varepsilon^{d / 2} f(\varepsilon x) .
$$

Our main results are stated in Theorems A, B and C below. In addition to (H-1), let

$$
\text { Hess } H_{m=0} \leq C \quad \text { for some positive constant } C \text {. }
$$

${ }^{1}$ By $\Lambda \uparrow \infty$, we mean that the size of box $\Lambda$ approaches $\infty$. 
Theorem A. Fix $\ell \in\{1, \ldots, d\}$. Then under hypotheses $(H-1)$ and $(H-2)$, the continuum scaling limit of the above models with $m=0$ is a continuum massless free field. In other words, there exists a constant positive definite matrix $q$ such that for all $f \in C_{0}^{\infty}\left(\mathbb{R}^{d}\right)$ and $t \in \mathbb{R}$,

$$
\lim _{\varepsilon \downarrow 0}\left\langle\mathrm{e}^{t \varphi\left(\nabla_{\ell}^{*} f_{\varepsilon}\right)}\right\rangle=\exp \left[\frac{t^{2}}{2}\left(f, \frac{-\partial}{\partial x_{\ell}} Q \frac{\partial}{\partial x_{\ell}} f\right)\right],
$$

where

$$
Q^{-1}=-\sum_{i, j=1}^{d} q_{i j} \frac{\partial}{\partial x_{i}} \frac{\partial}{\partial x_{j}}
$$

and $\varphi\left(\nabla_{\ell}^{*} f_{\varepsilon}\right)$ is defined in (1.2).

Remark 1. The RHS of Eq.(1.3) is the generating function of the continuum Gaussian probability measure with covariance $Q$.

Remark 2. It follows easily from our proof that if the model is (statistically) rotational invariant under lattice rotations, the continuum limit will have the full rotational invariance, that is $q$ will be a multiple of the identity.

Remark 3. For $m=0$, all these models enjoy the formal continuous symmetry $H(\varphi)=$ $H(\varphi+$ const.). In the presence of the mass term, this formal symmetry will be broken and the scaling limit will be a white noise Gaussian. In this case, the appropriate scaling is different from the one given above. For massive FKG models, a result due to Newman [11] proves the convergence to white noise. Note that in the nearest neighbor case, our conditions are sufficient to imply the FKG property.

Theorem B. Let $m>0$. Then there is a constant $m^{\prime}>0$ such that

$$
\left|\langle\varphi(x) \varphi(y)\rangle_{m}\right| \leq C \mathrm{e}^{-m^{\prime}\|x-y\|}, \text { for some } C>0 .
$$

Here $\langle\cdot\rangle_{m}$ denotes the expectation with respect to the infinite volume massive probability measure (with mass $m$ ), defined in (1.1).

For the massless model, we prove

Theorem C. Let $m=0$. Then for the above models in $d>2$, we have the following estimate:

$$
|\langle\varphi(x) \varphi(y)\rangle| \leq C\|x-y\|^{2-d}, \quad \text { for some } C>0 .
$$

Remark 4. We can relax the nearest neighbor restriction on the Hamiltonian. For any finite range interaction, Theorems A, B and C hold. See Sect. 4 for more details.

Remark 5. Theorems B and C will be proved separately from Theorem A. For simplicity, Theorem $\mathrm{C}$ has been used to prove Theorem $\mathrm{A}$ in $d>2$, however that could be also avoided (see the proof of Theorem $\mathrm{A}$ in $d=2$ ).

1.2. Notations. We shall always denote by $d$ the dimension of space. $\mathbb{Z}^{d}$ is the $d$ dimensional integral lattice and by $\mathbb{Z}_{\varepsilon}^{d}$, we mean the set

$$
\left\{\left(x_{1}, \ldots, x_{d}\right): x_{i} / \varepsilon \in \mathbb{Z}, i=1, \ldots, d\right\} .
$$


We always think of $\mathbb{Z}_{\varepsilon}^{d}$ as imbedded in $\mathbb{R}^{d}$ with coordinates which are integer multiples of $\varepsilon$, with respect to a Cartesian system defined by the unit vectors $\left\{\boldsymbol{e}_{i}: i=1, \ldots, d\right\}$. For any $x \in \mathbb{R}^{d},\|x\|$ will denote the Euclidean length of $x$.

For a real (or complex) valued function $f(x)$ on $\mathbb{R}^{d}$, and $i=1, \ldots, d$, define

$$
\begin{aligned}
& \left(\nabla_{i}^{\varepsilon} f\right)(x) \stackrel{\text { def. }}{=} \frac{f\left(x+\varepsilon e_{i}\right)-f(x)}{\varepsilon}, \\
& \left(\nabla_{i}^{\varepsilon *} f\right)(x) \stackrel{\text { def. }}{=} \frac{f\left(x-\varepsilon \boldsymbol{e}_{i}\right)-f(x)}{\varepsilon} .
\end{aligned}
$$

Note that $\nabla^{\varepsilon *}$ is the adjoint of $\nabla^{\varepsilon}$ with respect to the standard inner product of $\ell^{2}\left(\mathbb{Z}_{\varepsilon}^{d}\right)$. When $\varepsilon=1$, we shall drop the superscript $\varepsilon$. The discrete Laplacian (on $\mathbb{Z}^{d}$ ) will be denoted by $\Delta$.

Using the above notation, our (formal) Hamiltonian can be rewritten as

$$
H_{\lambda, m}(\varphi)=\sum_{x \in \mathbb{Z}^{d}} \sum_{i=1}^{d} V\left(\nabla_{i} \varphi(x)\right)+\frac{1}{2} m^{2} \sum_{x \in \mathbb{Z}^{d}} \varphi^{2}(x) .
$$

Let $\mu$ be the infinite volume translation invariant massless ergodic probability measure which was constructed in Eq.(1.1). We denote the expectation of a functional $u$ w.r.t. this measure by any of the following notations:

$$
\int u(\varphi) d \mu(\varphi) \equiv\langle u\rangle \equiv \boldsymbol{E}\{u\}
$$

Let $L^{2}(\mu)$ be the Hilbert space with inner product $(u, v)_{\mu} \stackrel{\text { def. }}{=}\langle u \bar{v}\rangle$. For a smooth functional $u(\varphi)$, we define

$$
\partial_{x} u(\varphi) \stackrel{\text { def. }}{=} \frac{\partial u(\varphi)}{\partial \varphi(x)}, \quad \partial_{x}^{*} u(\varphi) \stackrel{\text { def. }}{=}-\frac{\partial u(\varphi)}{\partial \varphi(x)}+\frac{\partial H(\varphi)}{\partial \varphi(x)} .
$$

Note that $\partial^{*}$ is the (formal) adjoint of $\partial$ with respect to the inner product:

$$
\left(\partial_{x}^{*} u, v\right)_{\mu}=\left(u, \partial_{x} v\right)_{\mu} .
$$

We define:

$$
-\Delta_{\varphi} \stackrel{\text { def. }}{=} \sum_{x \in \mathbb{Z}^{d}} \partial_{x}^{*} \partial_{x}
$$

For the entries of Hessian, we use the following short notation:

$$
H_{x y}^{\prime \prime} \stackrel{\text { def. }}{=} \partial_{x y}^{2} H \equiv \frac{\partial^{2} H}{\partial \varphi(x) \partial \varphi(y)} .
$$

We put,

$$
\sum_{x}^{\varepsilon} \stackrel{\text { def. }}{=} \varepsilon^{d} \sum_{x \in \mathbb{Z}_{\varepsilon}^{d}}
$$

and as a rule, we drop the superscript $\varepsilon$ whenever $\varepsilon=1$.

To simplify the notations, we shall use

$$
\left.a_{i}(x, \varphi) \stackrel{\text { def. }}{=} \frac{d^{2} V(z)}{d z^{2}}\right|_{z=\nabla_{i} \varphi(x)} .
$$


Then a simple calculation shows that $H^{\prime \prime}$ is a finite difference elliptic operator:

$$
\left(H^{\prime \prime} u\right)(x, \varphi)=\sum_{y} H_{x y}^{\prime \prime} u(y, \varphi)=\sum_{i=1}^{d} \nabla_{i}^{*}\left(a_{i}(x, \varphi) \nabla_{i} u(x, \varphi)\right) .
$$

Hypotheses (H-1) and (H-2) are then equivalent to

$$
0<a_{0}^{-1} \leq a_{i}(x, \varphi) \leq a_{0},
$$

for some constant $a_{0}>0$, uniformly in $\varphi$ and $x$.

Finally we warn the reader that the constants which will appear in different places are not necessarily equal, although we might use the same symbol for them.

1.3. Sketch of proofs. To prove our main results, we will use a representation which was originally introduced by Helffer and Sjöstrand $[8,12]$, in a somewhat different but related context. Fix $\ell \in\{1, \ldots, d\}$ and $f \in C_{0}^{\infty}\left(\mathbb{R}^{d}\right)$. We seek a solution $v_{\varepsilon}(y, \varphi), y \in$ $\mathbb{Z}^{d}$, to the following PDE:

$$
\sum_{y} \partial_{y}^{*} v_{\varepsilon}(y, \varphi)=\varphi\left(\nabla_{\ell}^{*} f_{\varepsilon}\right),
$$

in the space of gradient functionals, i.e. we assume $\partial_{y} v_{\varepsilon}(x, \varphi)=\partial_{x} v_{\varepsilon}(y, \varphi)$. By taking another derivative $\partial_{x}$ of the above PDE, and using this property, together with the observation that the commutator

$$
\left[\partial_{x}, \partial_{y}^{*}\right]=H_{x y}^{\prime \prime},
$$

we obtain:

$$
\begin{aligned}
\partial_{x} \varphi\left(\nabla_{\ell}^{*} f_{\varepsilon}\right) & =\partial_{x} \sum_{y} \partial_{y}^{*} v_{\varepsilon}(y, \varphi) \\
& =\sum_{y}\left(\partial_{y}^{*} \partial_{x}+\left[\partial_{x}, \partial_{y}^{*}\right]\right) v_{\varepsilon}(y, \varphi) \\
& =\sum_{y} \partial_{y}^{*} \partial_{x} v_{\varepsilon}(y, \varphi)+\sum_{y} H_{x y}^{\prime \prime} v_{\varepsilon}(y, \varphi) \\
& =\sum_{y} \partial_{y}^{*} \partial_{y} v_{\varepsilon}(x, \varphi)+\sum_{y} H_{x y}^{\prime \prime} v_{\varepsilon}(y, \varphi) .
\end{aligned}
$$

Therefore $v_{\varepsilon}$ satisfies the following PDE:

$$
\left(\mathscr{L} v_{\varepsilon}\right)(x, \varphi) \stackrel{\text { def. }}{=}-\Delta_{\varphi} v_{\varepsilon}(x, \varphi)+\sum_{i=1}^{d} \nabla_{i}^{*}\left(a_{i} \nabla_{i} v_{\varepsilon}\right)(x, \varphi)=\nabla_{\ell}^{*} f_{\varepsilon}(x)
$$

hence,

$$
v_{\varepsilon}=\left[-\Delta_{\varphi}+\sum_{i=1}^{d} \nabla_{i}^{*} a_{i} \nabla_{i}\right]^{-1} \nabla_{\ell}^{*} f_{\varepsilon}=\mathscr{L}^{-1} \nabla_{\ell}^{*} f_{\varepsilon}
$$


Using (1.8), we can express the pair correlation functions of $\varphi$ fields in terms of $v$ :

$$
\begin{aligned}
\left\langle\varphi\left(\nabla_{\ell}^{*} f_{\varepsilon}\right) \varphi\left(\nabla_{\ell}^{*} f_{\varepsilon}\right)\right\rangle & =\sum_{x}\left\langle\left(\partial_{x}^{*} v_{\varepsilon}(x, \varphi)\right) \varphi\left(\nabla_{\ell}^{*} f_{\varepsilon}\right)\right\rangle \\
& =\sum_{x}\left\langle v_{\varepsilon}(x, \varphi) \partial_{x} \varphi\left(\nabla_{\ell}^{*} f_{\varepsilon}\right)\right\rangle \\
& =\sum_{x}\left\langle v_{\varepsilon}(x, \varphi) \nabla_{\ell}^{*} f_{\varepsilon}(x)\right\rangle \\
& =\left\langle\left(\nabla_{\ell}^{*} f_{\varepsilon}, \mathscr{L}^{-1} \nabla_{\ell}^{*} f_{\varepsilon}\right)\right\rangle,
\end{aligned}
$$

where $($,$) denotes the standard inner product of \ell^{2}\left(\mathbb{Z}^{d}\right)$.

To prove Theorem A, we will show that $\left(\nabla_{\ell}^{*} f_{\varepsilon}, \mathscr{L}^{-1} \nabla_{\ell}^{*} f_{\varepsilon}\right) \rightarrow$ const. in $L^{2}(\mu)$; more precisely

$$
\left\langle\left|\left(\nabla_{\ell}^{*} f_{\varepsilon}, \mathscr{B}^{-1} \nabla_{\ell}^{*} f_{\varepsilon}\right)-Q_{f}\right|^{2}\right\rangle \rightarrow 0 \quad \text { as } \varepsilon \downarrow 0,
$$

where $Q_{f} \stackrel{\text { def. }}{=}\left(\nabla_{\ell}^{*} f_{\varepsilon}, Q \nabla_{\ell}^{*} f_{\varepsilon}\right)$ and

$$
Q^{-1}=-\sum_{i j} q_{i j} \frac{\partial^{2}}{\partial x_{i} \partial x_{j}}
$$

for the constant positive definite matrix $q$ given by

$$
q_{i j} \stackrel{\text { def. }}{=} \lim _{\beta \downarrow 0}\left\langle a_{i}\left(\delta_{i j}-\nabla_{i}(\mathscr{L}+\beta)^{-1} \nabla_{j}^{*} a_{j}\right)\right\rangle .
$$

Proof (of Theorem A, assuming (1.11)). Put

$$
G_{\varepsilon}(t) \stackrel{\text { def. }}{=}\left\langle\mathrm{e}^{t \varphi\left(\nabla_{\ell}^{*} f_{\varepsilon}\right)}\right\rangle \text {. }
$$

By the Brascamp-Lieb inequality, for any given $f$ there is a constant $C>0$, independent of $\varepsilon$ such that

$$
\left\langle\mathrm{e}^{t \varphi\left(\nabla_{\ell}^{*} f_{\varepsilon}\right)}\right\rangle \leq C .
$$

The constant $C$ can be chosen independent of $t$ for $t$ in any compact set. A straightforward calculation shows that

$$
\begin{aligned}
& \frac{d G_{\varepsilon}(t)}{d t}=t\left\langle\left(\sum_{x} v_{\varepsilon}(x, \varphi) \nabla_{\ell}^{*} f_{\varepsilon}(x)-Q_{f}\right) \mathrm{e}^{t \varphi\left(\nabla_{\ell}^{*} f_{\varepsilon}\right)}\right\rangle+ \\
& t Q_{f}\left\langle\mathrm{e}^{t \varphi\left(\nabla_{\ell}^{*} f_{\varepsilon}\right)}\right\rangle .
\end{aligned}
$$

Therefore

$$
\frac{d G_{\varepsilon}(t)}{d t}=t Q_{f} G_{\varepsilon}(t)+t A_{\varepsilon}(t),
$$

where $A_{\varepsilon}(t)=o(1)$ as $\varepsilon \downarrow 0$, by (1.11). Theorem A then follows upon integrating the last equality and then letting $\varepsilon \downarrow 0$. 
Remark 6. Following Helffer [7], the proof of B-L inequalities is a straightforward application of the above representation. To see this, consider $g(\varphi) \in C^{1} \cap L^{2}(\mu)$ and let $\sum_{x} \partial_{x} v(x, \varphi)=g-\langle g\rangle$, or equivalently $v=\mathscr{L}^{-1}(\partial g)$. Then a similar calculation to the one which yielded Eq.(1.10) gives

$$
\operatorname{Var} g=\left\langle\left(\partial g, \mathscr{B}^{-1} \partial g\right)\right\rangle \leq\left\langle\left(\partial g,\left(H^{\prime \prime}\right)^{-1} \partial g\right)\right\rangle,
$$

where the last inequality follows since $-\Delta_{\varphi} \geq 0$. This proves part (a) of B-L inequalities. Part (b) follows easily from part (a).

Remark 7. Instead of having an $L^{2}$ convergence in (1.11), any $L^{p}, p>1$ convergence would be sufficient. This might be useful if one wants to relax the hypothesis ( $\mathrm{H}-2)$.

The proof of (1.11) relies on homogenization. To see that, we first rescale as follows:

$$
\begin{aligned}
\sum_{x} v_{\varepsilon}(x, \varphi) \nabla_{\ell}^{*} f_{\varepsilon}(x) & =\varepsilon^{d} \sum_{x \in \mathbb{Z}_{\varepsilon}^{d}} u^{\varepsilon}(x, \varphi) \nabla_{\ell}^{\varepsilon *} f(x) \\
& =\sum_{x}^{\varepsilon} u^{\varepsilon}(x, \varphi) \nabla_{\ell}^{\varepsilon *} f(x),
\end{aligned}
$$

where $u^{\varepsilon}(x, \varphi) \stackrel{\text { def. }}{=} \varepsilon^{1-d / 2} v_{\varepsilon}(x / \varepsilon, \varphi), x \in \mathbb{Z}_{\varepsilon}^{d}$, satisfies

$$
\left(\mathscr{B}^{\varepsilon} u^{\varepsilon}\right)(x, \varphi) \stackrel{\text { def. }}{=}\left[\frac{-1}{\varepsilon^{2}} \Delta_{\varphi}+\sum_{i=1}^{d} \nabla_{i}^{\varepsilon *} a^{\varepsilon}(x / \varepsilon, \varphi) \nabla_{i}^{\varepsilon}\right] u^{\varepsilon}(x, \varphi)=\nabla_{\ell}^{\varepsilon *} f(x), x \in \mathbb{Z}_{\varepsilon}^{d} .
$$

If the first part of the above operator was absent, this equation could be viewed as a standard elliptic homogenization problem [15] (where $a_{i}(x / \varepsilon, \varphi)$ would play the role of the uniformly elliptic coefficients of an elliptic PDE, corresponding to the realization $\varphi$ of the random media). In Sect. 3 we show that suitable modifications of the standard techniques apply even in the presence of the first part of the operator and we obtain (1.11).

According to the above considerations, to obtain pointwise estimates on the two point functions, it suffices to obtain pointwise bounds on the kernel of $\mathscr{S}^{-1}$ (or $\left(\mathscr{C}+m^{2}\right)^{-1}$, for the massive case). Since $\mathscr{D}+m^{2} \geq m^{2}$, we can simply apply the Combes-Thomas' trick of conjugation and analytic continuation to prove Theorem B. This proof is quite straightforward and mainly uses the fact that $\mathscr{B}+m^{2}$, for $m>0$ is positive definite, therefore the result can be extended to a wider class of Hamiltonians. See Sect. 2.2.1 for details.

On the other hand, the massless case $(m=0)$ is not as easy as the massive $(m>0)$ case and uses the specific structure of the Hamiltonian. If we only had the second part of the operator $\mathscr{L}$, then the desired bounds would be a simple application of the Aronson estimates [3], which gives the following upper bound

$$
\Gamma\left(x, x^{\prime} ; t, 0\right) \leq C t^{-d / 2} \exp \left\{-\frac{\left\|x-x^{\prime}\right\|^{2}}{C t}\right\},
$$

on the fundamental solution $\Gamma$ of the problem

$$
\frac{\partial u(x, t)}{\partial t}=-\sum_{i=1}^{d} \nabla_{i}^{*} b_{i}(x, t) \nabla_{i} u(x, t)
$$


where

$$
0<a_{0}^{-1} \leq b_{i}(x, t) \leq a_{0} .
$$

In the proof of Theorem $\mathrm{C}$, we use the semi-group representation for the solution $v$ of (1.9). Upon trotterizing the corresponding semigroup, we observe that we are still able to use the Aronson upper bounds to get the desired estimate. Details are given in Sect. 2.2.2.

Note that Theorem B can be also proved by the same techniques as will be used in the proof of Theorem $\mathrm{C}$.

1.4. Organization. In Sect. 2, we mainly study Eqs.(1.8) and (1.9). After introducing the proper spaces and norms, we solve the second order PDE of (1.9) and then we show that its solution also satisfies (1.8). In the rest of that section we prove Theorems $B$ and $C$. Section 3 is devoted to the homogenization of the solution of (1.12). We shall see that the proof of homogenization follows from [15, 9] and relies mainly on Hilbert space methods and ergodicity. In Sect. 4, we will see that these results can be easily extended to long but finite range interactions. Finally, in the Appendix, we have collected some $\ell^{p}$ estimates which are used in Sect. 3.

\section{Helffer-Sjöstrand Representation and Proofs of Theorems B and C}

In this section, we study the Hellfer-Sjöstrand representation and related PDE's that will form the bases of our analysis. In Sect. 2.1, we give a rather careful definition of the relevant PDE's and the spaces in which we seek for solutions of them. We shall first show that Eq.(1.9), or equivalently Eq.(1.12) has a unique solution in the appropriate spaces. Then we verify that the solution of Eq.(1.9) satisfies Eq.(1.8) in the suitable sense (see Proposition 2.1). Finally in Sect. 2.2, we obtain some new bounds on the decay of two point functions, for both massive and massless models. These can be considered as pointwise extensions of the (form-wise) classical Brascamp-Lieb inequalities.

2.1. Some related PDE's and the Helffer-Sjöstrand representation. We first define the Hilbert space $\mathscr{H}^{1}(\mu)$ as the completion of the smooth local functionals under the inner product

$$
(u(\varphi), v(\varphi))_{\mathscr{X}} \stackrel{\text { def. }}{=}(u, v)_{\mu}+\sum_{x}\left(\partial_{x} u(\varphi), \partial_{x} v(\varphi)\right)_{\mu}
$$

When there is no danger of confusion, we will use the following shorthand:

$$
(\partial u, \partial v)_{\mu} \stackrel{\text { def. }}{=} \sum_{x}\left(\partial_{x} u(\varphi), \partial_{x} v(\varphi)\right)_{\mu}
$$

We also define the space $C_{0}\left(\mathbb{Z}_{\varepsilon}^{d}, \mathscr{B}^{1}\right)$ of compactly supported $\mathscr{B}^{1}(\mu)$-valued functionals.

For any $u, v \in C_{0}\left(\mathbb{Z}_{\varepsilon}^{d}, \mathscr{F}^{1}(\mu)\right)$, define the inner product

$$
(u, v)_{\mathfrak{D}_{\varepsilon}} \stackrel{\text { def. }}{=} \sum_{x}^{\varepsilon} \sum_{y}\left(\partial_{y} u(x, \varphi), \partial_{y} v(x, \varphi)\right)_{\mu}+\sum_{i=1}^{d} \sum_{x}^{\varepsilon}\left(\nabla_{i}^{\varepsilon} u, \nabla_{i}^{\varepsilon} v\right)_{\mu} .
$$


Completion of $C_{0}\left(\mathbb{Z}_{\varepsilon}^{d}, \mathscr{H}^{1}(\mu)\right)$ with respect to this inner product will be denoted by $\mathfrak{D}_{\varepsilon}^{1}\left(\mathbb{Z}_{\varepsilon}^{d}, \mu\right)$.

For fixed $f(x) \in C_{0}^{\infty}\left(\mathbb{R}^{d}\right), \varepsilon>0$ and $\ell \in\{1, \ldots, d\}$, consider the following problem:

$$
\left(\mathscr{L}^{\varepsilon} u\right)(x, \varphi) \stackrel{\text { def. }}{=} \frac{-1}{\varepsilon^{2}} \Delta_{\varphi} u(x, \varphi)+\sum_{i=1}^{d} \nabla_{i}^{\varepsilon *} a_{i}^{\varepsilon}(x, \varphi) \nabla_{i}^{\varepsilon} u(x, \varphi)=\nabla_{\ell}^{\varepsilon *} f(x),
$$

for $x \in \mathbb{Z}_{\varepsilon}^{d}$, where $a_{i}^{\varepsilon}(x, \varphi) \stackrel{\text { def. }}{=} a_{i}(x / \varepsilon, \varphi)$ satisfies (1.7).

We say $u \in \mathfrak{D}_{\varepsilon}^{1}\left(\mathbb{Z}_{\varepsilon}^{d}, \mu\right)$ is a (weak) solution to this problem if for every $v \in$ $\mathfrak{D}_{\varepsilon}^{1}\left(\mathbb{Z}_{\varepsilon}^{d}, \mu\right)$, we have

$$
\begin{aligned}
& \frac{1}{\varepsilon^{2}} \sum_{x}^{\varepsilon} \sum_{y}\left(\partial_{y} u(x, \varphi), \partial_{y} v(x, \varphi)\right)_{\mu}^{+} \\
& \quad \sum_{x}^{\varepsilon}\left(\nabla_{i}^{\varepsilon} u(x, \varphi), a_{i}^{\varepsilon}(x, \varphi) \nabla_{i}^{\varepsilon} v(x, \varphi)\right)_{\mu}=\sum_{x}^{\varepsilon}\left(f(x), \nabla_{\ell}^{\varepsilon} v(x, \varphi)\right)_{\mu} .
\end{aligned}
$$

The main tool to treat this type of PDE's is the Lax-Milgram theorem, which requires that the associated bilinear form be bounded and coercive (see [5]). Verifying these two conditions is straightforward and uses (1.7).

As we saw in the Introduction, the homogenization will be applied to the solution of the second order equation (2.1), while the statistical mechanical conclusions will be drawn using the solution of the first order equation (1.8). The next proposition identifies these two solutions. Let

$$
\begin{array}{r}
\mathscr{W} \stackrel{\text { def. }}{=}\left\{\text { all smooth local } w(\varphi) \text { s.t. } \sum_{x, y}\left\langle\left|\partial_{x y}^{2} w(\varphi)\right|^{2}\right\rangle+\sum_{x}\left\langle\left|\partial_{x} w(\varphi)\right|^{2}\right\rangle+\right. \\
\left.\left\langle|w(\varphi)|^{2}\right\rangle<\infty,\langle w\rangle=0\right\} .
\end{array}
$$

Proposition 2.1. Let $u \in \mathfrak{D}^{1}$ be the unique solution to (2.2), with $\varepsilon=1$. Then for any $w \in \mathscr{W}, \mathscr{W}$ defined in (2.3), we have

$$
\left(w, \sum_{x} \partial_{x}^{*} u(x, \varphi)-\varphi\left(\nabla_{\ell}^{\varepsilon} f\right)\right)_{\mu}=0 .
$$

Proof. Put $\varepsilon=1$. Let $u \in \mathfrak{D}^{1}\left(\mathbb{Z}^{d}, \mu\right)$ be the unique weak solution to the PDE

$$
\mathscr{L} u(x, \varphi)=\nabla_{\ell}^{*} f(x), \quad x \in \mathbb{Z}^{d}
$$

Assume that for all $x, y \in \mathbb{Z}^{d}, u$ is of gradient type, i.e.

$$
\left(v, \partial_{x} u(y, \varphi)\right)_{\mu}=\left(v, \partial_{y} u(x, \varphi)\right)_{\mu}, \quad \forall v \in L^{2}(\mu)
$$

Then, using this equality, the commutation relation and the definition of $u$,

$$
\left(\sum_{x} \partial_{x}^{*} v(x, \varphi), \sum_{y} \partial_{y}^{*} u(y, \varphi)-\varphi\left(\nabla_{\ell}^{*} f\right)\right)_{\mu}=0
$$


Proposition will follow if we show that the subspace

$$
\mathscr{V} \stackrel{\text { def. }}{=}\left\{\sum_{x} \partial_{x}^{*} v(x, \varphi): v(x, \varphi) \in \mathfrak{D}^{1}\right\}
$$

is dense in $\mathscr{W}$. To prove this, assume that $\exists w \in \mathscr{W}$ such that for all $v \in \mathscr{V}$,

$$
\left(\sum_{x} \partial_{x}^{*} v(x, \varphi), w(\varphi)\right)_{\mu}=0 .
$$

On the other hand, for $w \in \mathscr{W}, v(x, \varphi)=\partial_{x} w(\varphi) \in \mathfrak{D}^{1}$, therefore we can put $v(x, \varphi)=\partial_{x} w(\varphi)$ in the above equations to obtain

$$
\sum_{x}\left\langle\left|\partial_{x} w(\varphi)\right|^{2}\right\rangle=0
$$

which shows that $w=$ const., but the only constant in $\mathscr{W}$ is the zero vector so $w=0$. This proves that $\mathscr{V}$ is dense in $\mathscr{W}$.

Next we justify the assumption (A). Let

$$
C_{0}^{\text {grad }}\left(\mathbb{Z}^{d}, \mathscr{H} \mathscr{C}^{1}\right) \stackrel{\text { def. }}{=}\left\{u \in C_{0}\left(\mathbb{Z}^{d}, \mathscr{H} \mathscr{C}^{1}\right): u \text { satisfies (A) }\right\} .
$$

Then one can easily see that $\mathscr{B}$ leaves $C_{0}^{\text {grad }}$ invariant. If we solve $\mathscr{B} u=\nabla_{\ell}^{\varepsilon *} f$ in the completion of this space (which is a subspace of $\mathfrak{D}^{1}$ ), we will obtain a unique solution in this space. Note that $\nabla_{\ell}^{*} f \in C_{0}^{\text {grad }}\left(\mathbb{Z}^{d}, \mathscr{B}^{1}\right)$ since $\nabla_{\ell}^{*} f(x)=\partial_{x} \varphi\left(\nabla_{\ell}^{*} f\right)$. The earlier considerations about the existence and uniqueness of the solution in the larger space $\mathfrak{D}^{1}$ and the invariance of $C_{0}^{\text {grad }}$ under $\mathscr{B}$ imply that these two solutions should agree and this justifies the assumption (A) and finishes the proof.

Remark 8. Equation (2.4) can be extended to be valid for $w \in \mathscr{W}+$ const., since

$$
\left(\text { const. }, \sum_{x} \partial_{x}^{*} u(x, \varphi)-\varphi\left(\nabla_{\ell}^{*} f\right)\right)_{\mu}=0 .
$$

2.2. Some new pointwise bounds on the 2-point functions. In the first part of this section, we will consider the massive models, i.e. $m>0$. Bounds on the decay of massless models will be obtained in the second part. These bounds are the pointwise extensions of the Brascamp-Lieb inequalities and are comparable to the bounds that one can obtain for the pure Gaussian models.

2.2.1. Massive models. For a fixed $m>0$, consider the operator

$$
\mathscr{D}=-\Delta_{\varphi}+\sum_{i=1}^{d} \nabla_{i}^{*} a_{i} \nabla_{i}+m^{2},
$$

defined on $\ell^{2}\left(\mathbb{Z}^{d} ; \mathscr{H} \mathscr{C}^{1}(\mu)\right.$ ). Note that as a quadratic form, $\mathscr{B} \geq m^{2}$ (see Remark 9

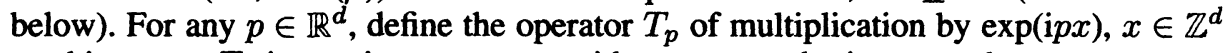
on this space. $T_{p}$ is a unitary operator with respect to the inner product 


$$
(u, v) \stackrel{\text { def. }}{=}\left\langle\sum_{x} u(x, \varphi) \overline{v(x, \varphi)}\right\rangle,
$$

with inverse $T_{p}^{-1}=T_{-p}$. Finally define the operator:

$$
\mathscr{L}_{p} \stackrel{\text { def. }}{=} T_{p}^{-1} \mathscr{D} T_{p}
$$

Since $T_{p}$ commutes with $-\Delta_{\varphi}$, it is easy to check that for small $|p|, p \in \mathbb{C}^{d}, \mathscr{D}_{p}$ is still positive definite and hence invertible.

Theorem B. There is a constant $m^{\prime}=m^{\prime}\left(a_{0}, m, d\right)>0$ such that

$$
\langle\varphi(x) \varphi(y)\rangle \leq C \mathrm{e}^{-m^{\prime}\|x-y\|}, \quad \text { for some } C>0 .
$$

Proof. The above considerations show that $\left(T_{p}^{-1} \mathscr{S} T_{p}\right)^{-1}=T_{p}^{-1} \mathscr{L}^{-1} T_{p}$, which was originally defined for $p \in \mathbb{R}^{d}$, has an analytic continuation into the disk $|p|<p_{0}$ of the complex plane. Therefore we can choose $m^{\prime}=m^{\prime}\left(a_{0}, m, d\right)$ such that

$$
\left|\mathscr{S}^{-1}\left(x, x^{\prime} ; \varphi, \varphi^{\prime}\right)\right| \leq C \mathrm{e}^{-m^{\prime}\left\|x-x^{\prime}\right\|},
$$

uniformly in $\varphi$ and $\varphi^{\prime}$.

Remark 9. The same argument, and hence result, holds if instead of having an $m>0$ explicitly in $\mathscr{L}$, we assume that $\mathscr{L} \geq m^{2}$; after all that is the only hypothesis we have used.

2.2.2. Massless models. We shall obtain a power law decay for the kernel of $\mathscr{L}^{-1}$, where

$$
\mathscr{C}=-\Delta_{\varphi}+\sum_{i=1}^{d} \nabla_{i}^{*} a_{i} \nabla_{i}=-\Delta_{\varphi}+H^{\prime \prime},
$$

where $a_{i}$ satisfy (1.7). Proof of Theorem $\mathrm{C}$ will then be a simple application of this result.

We start with the semigroup generated by this non-negative (self-adjoint) operator ${ }^{2}$. Since each of the two parts of $\mathscr{D}$ is non-negative, we have the following Trotter product representation for this semigroup:

$$
\mathrm{e}^{-t \mathscr{C}}=\lim _{n \rightarrow \infty}\left(\mathrm{e}^{t / n \Delta_{\varphi}} \mathrm{e}^{-t / n H^{\prime \prime}}\right)^{n}
$$

The above convergence takes place in the strong operator topology. Put

$$
\begin{aligned}
I_{t}^{(n)} & \stackrel{\text { def. }}{=}\left(\mathrm{e}^{t / n \Delta_{\varphi}} \mathrm{e}^{-t / n H^{\prime \prime}}\right)^{n} \\
& =\mathrm{e}^{t / n \Delta_{\varphi}} \mathrm{e}^{-t / n H^{\prime \prime}} \cdots \mathrm{e}^{t / n \Delta_{\varphi}} \mathrm{e}^{-t / n H^{\prime \prime}}, \quad \text { (n pairs) }
\end{aligned}
$$

and define the following kernels:

$$
\begin{array}{rll}
S_{t}(\varphi, \psi) & \stackrel{\text { def. }}{=} & \mathrm{e}^{t \Delta_{\varphi}}(\varphi, \psi), \\
k_{t}(x, y ; \varphi) \stackrel{\text { def. }}{=} & \mathrm{e}^{-t H^{\prime \prime}}(x, y ; \varphi) .
\end{array}
$$

${ }^{2}$ The non-negative quadratic form induced by $\mathscr{B}$ on $\mathfrak{D}^{1}$ gives a self-adjoint extension of $\mathscr{B}$ which we will again denote by $\mathscr{B}$. 
Therefore the kernel of $I_{t}^{(n)}$ can be written as

$$
\begin{aligned}
& I_{t}^{(n)}(x, y ; \varphi, \psi)=\sum_{x_{n-1}, \ldots, x_{1}} \int d \mu\left(\varphi^{n-1}\right) \ldots d \mu\left(\varphi^{1}\right) S_{t / n}\left(\varphi, \varphi^{n-1}\right) \\
& k_{t / n}\left(x, x_{n-1} ; \varphi^{n-1}\right) \cdots S_{t / n}\left(\varphi^{1}, \psi\right) k_{t / n}\left(x_{1}, y ; \psi\right) \\
&= \int d \mu\left(\varphi^{n-1}\right) \ldots d \mu\left(\varphi^{1}\right) S_{t / n}\left(\varphi, \varphi^{n-1}\right) \cdots S_{t / n}\left(\varphi^{1}, \psi\right) \times \\
& \sum_{x_{n-1}, \ldots, x_{1}} k_{t / n}\left(x, x_{n-1} ; \varphi^{n-1}\right) \cdots k_{t / n}\left(x_{1}, y ; \psi\right) .
\end{aligned}
$$

For fixed $\varphi, \varphi^{1}, \ldots, \varphi^{n-1}, \psi$, let

$$
u^{(n)}(t, x) \stackrel{\text { def. }}{=} \sum_{x_{n-1}, \ldots, x_{1}, y} k_{t / n}\left(x, x_{n-1} ; \varphi^{n-1}\right) \cdots k_{t / n}\left(x_{1}, y ; \psi\right) f(y) .
$$

Then it is clear that for fixed $n$ and $t, u^{(n)}(t, x)$ is the solution at time $s=t$ of the following problem:

$$
\left\{\begin{array}{l}
\partial u^{(n)}(s, x) / \partial s=-\sum_{i=1}^{d} \nabla_{i}^{*} b_{i}^{(n)}(x, s) \nabla_{i} u^{(n)}(s, x) \\
u^{(n)}(0, x)=f(x)
\end{array}\right.
$$

where

$$
b_{i}^{(n)}(x, s) \stackrel{\text { def. }}{=} a_{i}\left(x, \varphi^{k}\right) \quad \text { for } \quad(k-1) \frac{t}{n} \leq s<k \frac{t}{n}, \quad k=1, \ldots, n .
$$
time $t$ :

Back to Eq.(2.5), we know that its solution has the following representation at

$$
u^{(n)}(t, x)=\sum_{y} \Gamma^{(n)}(t, 0 ; x, y) f(y),
$$

where $\Gamma^{(n)}(t, s ; x, y)$ is the fundamental solution corresponding to Eq.(2.5), with the initial condition $f(x)=\delta_{x y}$. Since $b_{i}(x, t)$ is bounded from above and below (uniformly in $n, x$ and $t$ ), Aronson's estimate [3] implies the existence of a constant $C$ (which only depends on dimension $d$ and the ellipticity constant $a_{0}$ ) such that

$$
\Gamma^{(n)}(t, 0 ; x, y) \leq \frac{C}{t^{d / 2}} \mathrm{e}^{-\|x-y\|^{2} / C t},
$$

which, in turn, gives us the following bound:

$$
\sum_{x_{n-1}, \ldots, x_{1}} k_{t / n}\left(x, x_{n-1} ; \varphi^{n-1}\right) \cdots k_{t / n}\left(x_{1}, y ; \psi\right) \leq \frac{C}{t^{d / 2}} \mathrm{e}^{-\|x-y\|^{2} / C t}
$$

uniformly in $\varphi$ 's.

Since the operator $\exp \left\{t \Delta_{\varphi}\right\}$ preserves positivity, and hence its kernel $S_{t}(\varphi, \psi)$ is nonnegative, we can estimate the kernel of $I_{t}^{(n)}$ through the above estimate. Putting $S_{t / n}$ 's together, we obtain:

$$
I_{t}^{(n)}(x, y ; \varphi, \psi) \leq C t^{-d / 2} \mathrm{e}^{-\|x-y\|^{2} / C t} \mathrm{e}^{t \Delta_{\varphi}}(\varphi, \psi) .
$$

Since

$$
\langle\varphi(x) \varphi(y)\rangle=\int \mathscr{D}^{-1}(x, y ; \varphi, \psi) d \mu(\varphi) d \mu(\psi),
$$

Theorem $\mathrm{C}$ follows by integrating out $t$ in the above estimate and then letting $n \rightarrow \infty$. 


\section{Homogenization and the Proof of Theorem A}

In this section, we shall extend the standard techniques of homogenization (see [15, $9,6])$ to the elliptic PDE (1.12) to prove Theorem A. Throughout this section, we will use the massless probability measure constructed in (1.1). Since the whole proof is rather lengthy, we will break it into two steps which we will describe here briefly.

Step 1. This step is the heart of the proof of Theorem A. Fix $\lambda>0 ; \ell \in\{1, \ldots, d\}$ and $f \in C_{0}^{\infty}\left(\mathbb{R}^{d}\right)$, and consider the following PDE:

$$
\left(\mathscr{D}^{\varepsilon}+\lambda\right) u_{\lambda}^{\varepsilon}(x, \varphi)=\nabla_{\ell}^{\varepsilon *} f(x), \quad x \in \mathbb{Z}_{\varepsilon}^{d} .
$$

Let $w_{\lambda}^{\varepsilon}(x)$ satisfies

$$
\left(\sum_{i, j=1}^{d} q_{i j} \nabla_{i}^{\varepsilon *} \nabla_{j}^{\varepsilon}+\lambda\right) w_{\lambda}^{\varepsilon}(x)=\nabla_{\ell}^{\varepsilon *} f(x), \quad x \in \mathbb{Z}_{\varepsilon}^{d} .
$$

The key step is to show that the constant positive definite matrix $q$ can be chosen so that $\left\|u_{\lambda}^{\varepsilon}-w_{\lambda}^{\varepsilon}\right\|_{\varepsilon} \rightarrow 0$ as $\varepsilon \downarrow 0$ (see Theorem 3.1). We construct the constant effective diffusion matrix $q$ by introducing an auxiliary function $\chi_{i}^{\beta}$ which formally satisfies (cf. (3.7))

$$
(\mathscr{B}+\beta) \chi_{i}^{\beta}=-\nabla_{i}^{*} a_{i}
$$

As $\beta \downarrow 0, \nabla_{i} \chi_{j}^{\beta}$ has a well defined limit, which will be denoted by $\psi_{i j}$. A very important observation is that $\beta E\left(\chi_{i}^{\beta}\right)^{2} \rightarrow 0$ as $\beta \downarrow 0$ (see Proposition 3.1). We then define

$$
q_{i j}=\boldsymbol{E} a_{i}\left(\delta_{i j}+\psi_{i j}\right),
$$

and introduce the error function

$$
z^{\varepsilon}(x, \varphi)=u^{\varepsilon}(x, \varphi)-w^{\varepsilon}(x)+\varepsilon \sum_{i=1}^{d} \chi_{i}^{\varepsilon^{2}}(x / \varepsilon, \varphi) \nabla_{i}^{\varepsilon *} w^{\varepsilon}(x) .
$$

As we just mentioned, the last term in the error function will go to zero as $\varepsilon \downarrow 0$, therefore to prove that $\left\|u_{\lambda}^{\varepsilon}-w_{\lambda}^{\varepsilon}\right\|_{\varepsilon} \rightarrow 0$, it suffices to prove that $\left\|z^{\varepsilon}\right\|_{\varepsilon} \rightarrow 0$. One way of doing this is to calculate $\left(z^{\varepsilon},\left(\mathscr{L}^{\varepsilon}+\lambda\right) z^{\varepsilon}\right)_{\varepsilon}$ and show that this expression vanishes. Since $\mathscr{L}^{\varepsilon}+\lambda$ is uniformly elliptic, this last statement implies $\left\|z^{\varepsilon}\right\|_{\varepsilon} \rightarrow 0$. These will be our plans for Step 1. It is a standard result that as $\varepsilon \downarrow 0,\left\|w_{\lambda}^{\varepsilon}-w_{\lambda}\right\|_{\varepsilon} \rightarrow 0$, where $w_{\lambda}$ satisfies

$$
\left(-\sum_{i, j} q_{i j} \frac{\partial^{2}}{\partial x_{i} \partial x_{j}}+\lambda\right) w_{\lambda}(x)=-\frac{\partial f}{\partial x_{\ell}}, \quad x \in \mathbb{R}^{d} .
$$

As a result, in this step we show that for $\lambda>0,\left\|u_{\lambda}^{\varepsilon}-w_{\lambda}\right\|_{\varepsilon} \rightarrow 0$, as $\varepsilon \downarrow 0$. We call this step the massive homogenization due to the presence of the positive constant $\lambda>0$, which plays the role of a mass. The main reason for adding this mass (which will be later removed in Step 2) is that we are solving these PDE's in the whole space and in the presence of such a mass, solutions have exponentially fast decay at infinity. We remark that the probability measure is kept massless. 
Step 2. We extend the results of Step 1 to the massless case $\lambda=0$. Namely, in this step we show that for the solutions $u_{\lambda}^{\varepsilon}$ and $w_{\lambda}$ of (3.1) and (3.2), and any $\ell^{\prime} \in\{1$, ldots, $d\}$, we have, as $\lambda \downarrow 0$,

$$
\begin{aligned}
\sum_{x}^{\varepsilon} \nabla_{\ell^{\prime}}^{\varepsilon *} g(x) u_{\lambda}^{\varepsilon}(x, \varphi) & \rightarrow \sum_{x}^{\varepsilon} \nabla_{\ell^{\prime}}^{\varepsilon *} g(x) u_{0}^{\varepsilon}(x, \varphi), \\
\sum_{x}^{\varepsilon} \nabla_{\ell^{\prime}}^{\varepsilon *} g(x) w_{\lambda}(x) & \rightarrow \sum_{x}^{\varepsilon} \nabla_{\ell^{\prime}}^{\varepsilon *} g(x) w_{0}(x),
\end{aligned}
$$

uniformly in $\varepsilon$, where $u_{0}^{\varepsilon}$ and $w_{0}$ satisfy Eqs. (3.1) and (3.2) with $\lambda=0$. This and the result of Step 1 will prove that

$$
\sum_{x}^{\varepsilon} \nabla_{\ell^{\prime}}^{\varepsilon *} g(x)\left[u_{0}^{\varepsilon}(x, \varphi)-w_{0}(x)\right] \stackrel{L^{2}(\mu)}{\longrightarrow} 0, \quad \text { as } \varepsilon \downarrow 0 .
$$

We know from Sect. 1.3 that this last statement implies Theorem A.

3.1. Step 1: Massive homogenization. For $x \in \mathbb{Z}^{d}$, define $T_{x}: L^{2}(\mu) \rightarrow L^{2}(\mu)$ by

$$
\left(T_{x} \widetilde{g}\right)(\varphi)=\widetilde{g}\left(\tau_{-x} \varphi\right),
$$

where $\left(\tau_{x} \varphi\right)(y)=\varphi(y-x), x, y \in \mathbb{Z}^{d}$. The $\tau$-invariance of $\mu$ (i.e. the translation invariance of $\mu$ ) shows that $\left\{T_{x}\right\}_{x \in \mathbb{Z}^{d}}$ is a family of unitary transformations on $L^{2}(\mu)$. Since the translation group $\tau_{x}, x \in \mathbb{Z}^{d}$ is ergodic, the only functions in $L^{2}(\mu)$ that are invariant under the unitary group $\left\{T_{x}, x \in \mathbb{Z}^{d}\right\}$, are constant functions. Let $D_{1}, \cdots, D_{d}$ denote the generators of this unitary group along the directions $e_{1}, \ldots, e_{d}$ :

$$
\left(D_{i} \widetilde{g}\right)(\varphi) \stackrel{\text { def. }}{=}\left(T_{e_{i}} \widetilde{g}\right)(\varphi)-\widetilde{g}(\varphi),
$$

and let $D_{i}^{*}$ be the adjoint of $D_{i}$, with respect to $(,)_{\mu}$, that is $\left(\widetilde{g}, D_{i} \tilde{h}\right)_{\mu}=$ $\left(D_{i}^{*} \widetilde{g}, \widetilde{h}\right)_{\mu}$.

We define the inner product ${ }^{3}$ :

$$
(\widetilde{g}, \widetilde{h})_{1, \mu} \stackrel{\text { def. }}{=}(\widetilde{g}, \widetilde{h})_{\mu}+\sum_{i=1}^{d}\left(D_{i} \widetilde{g}, D_{i} \widetilde{h}\right)_{\mu}+(\partial \widetilde{g}, \partial \tilde{h})_{\mu}
$$

on the Hilbert space $\mathscr{H}^{1}(\mu)$. Corresponding to any $\widetilde{g} \in L^{2}(\mu)$, we define a stationary $g$ by

$$
g(x, \varphi) \stackrel{\text { def. }}{=}\left(T_{x} \widetilde{g}\right)(\varphi)=\widetilde{g}\left(\tau_{-x} \varphi\right), \quad x \in \mathbb{Z}^{d} .
$$

$\tau$-invariance of $\mu$ implies that the expectation of any such function is independent of $x$, and we also have

$$
\nabla_{i} g(x, \varphi)=D_{i} g(x, \varphi), \quad \nabla_{i}^{*} g(x, \varphi)=D_{i}^{*} g(x, \varphi)
$$

for such functions.

Define the Hilbert space $H_{\varepsilon}\left(\mathbb{Z}_{\varepsilon}^{d}, \mu\right) \stackrel{\text { def. }}{=} \ell^{2}\left(\mathbb{Z}_{\varepsilon}^{d} ; L^{2}(\mu)\right)$ of square summable functions over $\mathbb{Z}_{\varepsilon}^{d}$ with values in $L^{2}(\mu)$, with inner product

\footnotetext{
${ }^{3}$ Note that the norm induced by this inner product is equivalent to the one induced by $(,)_{\mathscr{H}}$ 1.
} 


$$
\begin{gathered}
(u, v)_{\varepsilon} \stackrel{\text { def. }}{=} \boldsymbol{E} \varepsilon^{d} \sum_{x \in \mathbb{Z}_{\varepsilon}^{d}} u(x, \varphi) \overline{v(x, \varphi)} \equiv \boldsymbol{E} \sum_{x}^{\varepsilon} u(x, \varphi) \overline{v(x, \varphi)} \\
\|u\|_{\varepsilon}^{2}=(u, u)_{\varepsilon} .
\end{gathered}
$$

Finally define the Hilbert space $H_{\varepsilon}^{1}\left(\mathbb{Z}_{\varepsilon}^{d}, \mu\right) \stackrel{\text { def. }}{=} H_{\varepsilon}^{1}\left(\mathbb{Z}_{\varepsilon}^{d} ; \mathscr{F}^{1}(\mu)\right)$ of square summable $\mathscr{H}^{1}(\mu)$-valued functions over $\mathbb{Z}_{\varepsilon}^{d}$ with the inner product:

$$
\begin{aligned}
&(u, v)_{1, \varepsilon} \stackrel{\text { def. }}{=}(\partial u, \partial v)_{\varepsilon}+\sum_{i=1}^{d}\left(\nabla_{i}^{\varepsilon} u, \nabla_{i}^{\varepsilon} v\right)_{\varepsilon}+(u, v)_{\varepsilon} \\
& \equiv E\left(\varepsilon^{d} \sum_{y} \sum_{x \in \mathbb{Z}_{\varepsilon}^{d}} \partial_{y} u(x, \varphi) \partial_{y} \overline{v(x, \varphi)}\right)+ \\
&+E\left(\varepsilon^{d} \sum_{i=1}^{d} \sum_{x \in \mathbb{Z}_{\varepsilon}^{d}} \nabla_{i} u(x, \varphi) \nabla_{i} \overline{v(x, \varphi)}\right)+ \\
&+E\left(\varepsilon^{d} \sum_{x \in \mathbb{Z}_{\varepsilon}^{d}} u(x, \varphi) \overline{v(x, \varphi)}\right)
\end{aligned}
$$

and

$$
\|u\|_{1, \varepsilon}^{2} \stackrel{\text { def. }}{=}(u, u)_{1, \varepsilon} \text {. }
$$

Fix $\lambda>0$ and $\ell \in\{1, \ldots, d\}$. For simplicity, we shall drop the subscript $\lambda$ in this section. For $f \in C_{0}^{\infty}\left(\mathbb{R}^{d}\right)$, let $u^{\varepsilon}(x, \varphi) \in H_{\varepsilon}^{1}\left(\mathbb{Z}_{\varepsilon}^{d}, \mu\right)$ be the solution to

$$
\frac{1}{\varepsilon^{2}}\left(\partial u^{\varepsilon}, \partial v\right)_{\varepsilon}+\sum_{i=1}^{d}\left(a_{i}^{\varepsilon} \nabla_{i}^{\varepsilon} u^{\varepsilon}, \nabla_{i}^{\varepsilon} v\right)_{\varepsilon}+\lambda(u, v)_{\varepsilon}=\left(f, \nabla_{\ell}^{\varepsilon} v\right)_{\varepsilon}
$$

for all $v \in H_{\varepsilon}^{1}\left(\mathbb{Z}_{\varepsilon}^{d}, \mu\right)$, where $a_{i}^{\varepsilon}(x, \varphi) \equiv a_{i}(x / \varepsilon, \varphi), x \in \mathbb{Z}_{\varepsilon}^{d}$. By translation invariance, $a_{i}(x, \varphi)=T_{x} \widetilde{a}_{i}(\varphi)$ for some $\tilde{a}_{i}(\varphi) \in \mathscr{H}^{1}(\mu)$ and there exists a constant $a_{0}>0$ such that

$$
a_{0}^{-1} \leq a_{i}(x, \varphi) \leq a_{0}, \quad \text { uniformly in } x, \varphi .
$$

Using this, it is a quite straightforward application of the Lax-Milgram theorem to establish the existence and uniqueness of the solution to this PDE. We further obtain the following estimate:

$$
\|u\|_{1, \varepsilon}^{2} \leq C \int f^{2}(x) d x, \quad \text { uniformly in } \varepsilon .
$$

The (lattice) homogenized problem is the following. Let $H_{\varepsilon}^{1}\left(\mathbb{Z}_{\varepsilon}^{d}\right)$ be the Hilbert space of all square summable functions $u$ on $\mathbb{Z}_{\varepsilon}^{d}$ with square summable derivatives, with inner product

$$
(u, v)_{1, \varepsilon}=\sum_{x}^{\varepsilon} u(x) \overline{v(x)}+\sum_{x}^{\varepsilon} \sum_{i} \nabla_{i}^{\varepsilon} u(x) \nabla_{i}^{\varepsilon} \overline{v(x)} .
$$


Note that we have used the same notation for the inner product on $H_{\varepsilon}^{1}\left(\mathbb{Z}_{\varepsilon}^{d}, \mu\right)$ and $H_{\varepsilon}^{1}\left(\mathbb{Z}_{\varepsilon}^{d}\right)$ since they agree on the functions which only depend on $x$. For $f \in C_{0}^{\infty}\left(\mathbb{R}^{d}\right)$, let $w^{\varepsilon} \in H_{\varepsilon}^{1}\left(\mathbb{Z}_{\varepsilon}^{d}\right)$ satisfies

$$
\sum_{x}^{\varepsilon} \sum_{i, j=1}^{d} q_{i j} \nabla_{i}^{\varepsilon} w^{\varepsilon}(x) \nabla_{j}^{\varepsilon} \overline{v(x)}+\lambda \sum_{x}^{\varepsilon} w^{\varepsilon}(x) \overline{v(x)}=\sum_{x}^{\varepsilon} f(x) \nabla_{\ell}^{\varepsilon} \overline{v(x)},
$$

for all $v \in H_{\varepsilon}^{1}\left(\mathbb{Z}_{\varepsilon}^{d}\right)$. Here we are assuming that $q_{i j}$ is a positive definite symmetric matrix (see Lemma (3.1)). Existence and uniqueness of the solution again follows easily from the Lax-Milgram theorem. Furthermore, since $f \in C_{0}^{\infty}\left(\mathbb{R}^{d}\right)$, the solution $w^{\varepsilon}$ goes to zero exponentially fast as $|x| \rightarrow \infty$ for $\lambda>0$.

We would like to show that for suitably defined $q_{i j}$, the solution $u^{\varepsilon}$ of Eq.(3.3) approaches as $\varepsilon \downarrow 0$ to the solution $w^{\varepsilon}$ of Eq.(3.6), in a suitable sense.

Theorem 3.1. For $f \in C_{0}^{\infty}\left(\mathbb{R}^{d}\right), \lambda>0$, let $u^{\varepsilon} \in H_{\varepsilon}^{1}\left(\mathbb{Z}_{\varepsilon}^{d}, \mu\right)$ be the solution to Eq.(3.3). Then there is a positive definite constant matrix $q_{i j}$ such that

$$
\left\|u^{\varepsilon}(x, \varphi)-w^{\varepsilon}(x)\right\|_{\varepsilon} \rightarrow 0 \quad \text { as } \varepsilon \downarrow 0,
$$

where $w^{\varepsilon}(x) \in H^{1}\left(\mathbb{Z}_{\varepsilon}^{d}\right)$ is the solution of $E q .(3.6)$.

The first step towards the proof is the construction of $q_{i j}$. To this end, we consider the following problem. For any constant $\beta>0$, let $\tilde{\chi}_{i}^{\beta} \in \mathscr{H}^{1}(\mu), i=1, \cdots, d$, be the solution to

$$
\left(\partial \widetilde{v}, \partial \widetilde{\chi}_{i}^{\beta}\right)_{\mu}+\sum_{j=1}^{d}\left(D_{j} \widetilde{v}, \tilde{a}_{j} D_{j} \widetilde{\chi}_{i}^{\beta}\right)_{\mu}+\beta\left(\widetilde{v}, \tilde{\chi}_{i}^{\beta}\right)_{\mu}=-\left(\tilde{a}_{i}, D_{i} \widetilde{v}\right)_{\mu}
$$

for all $\widetilde{v} \in \mathscr{H}^{1}(\mu)$. Coercivity and boundedness of the corresponding bilinear form follows easily, which, upon using the Lax-Milgram theorem, implies the existence and uniqueness of the solution. Moreover one can easily obtain the following estimates. For all $i, j=1, \ldots, d$,

(a) $\beta \boldsymbol{E}\left\{\left(\tilde{\chi}_{i}^{\beta}\right)^{2}\right\} \leq C$,

(b) $\boldsymbol{E}\left(D_{j} \widetilde{\chi}_{i}^{\beta}\right)^{2} \leq C$,

(c) $\boldsymbol{E}\left(\boldsymbol{\partial} \widetilde{\chi}_{i}^{\beta}\right)^{2} \leq C$.

The constants on the RHS's do not depend on $\beta$. Because of estimates (b) and (c), there is a subsequence $\beta^{\prime} \downarrow 0$ along which

$$
\begin{array}{ll}
D_{j} \widetilde{\chi}_{i}^{\beta^{\prime}} \rightarrow \widetilde{\psi}_{j i} & \text { (some limit) } \\
\partial_{x} \widetilde{\chi}_{i}^{\beta^{\prime}} \rightarrow \widetilde{\xi}_{x, i} & \text { (some limit) }
\end{array}
$$

weakly in $L^{2}(\mu)$. Using the estimate (a) we can pass to the limit in Eq.(3.7) along that subsequence to obtain

$$
\sum_{x}\left(\partial_{x} \tilde{v}, \tilde{\xi}_{x, i}\right)_{\mu}+\sum_{j=1}^{d}\left(D_{j} \widetilde{v}, \tilde{a}_{j} \widetilde{\psi}_{j i}\right)_{\mu}=-\left(\tilde{a}_{i}, D_{i} \widetilde{v}\right)_{\mu} .
$$

We further notice that the limits in (3.8), are independent of the subsequence. To see this, assume that we would obtain the limits $\{\xi, \psi\}$ and $\left\{\xi^{\prime}, \psi^{\prime}\right\}$ as $\beta \downarrow 0$ along the 
subsequences $\beta^{(1)}$ and $\beta^{(2)}$, respectively. Then, for all $\widetilde{v} \in \mathscr{H}^{1}(\mu), \bar{\xi}=\xi-\xi^{\prime}$ and $\bar{\psi}=\psi-\psi^{\prime}$ satisfy

$$
\sum_{x}\left(\partial_{x} \tilde{v}, \bar{\xi}_{x, i}\right)_{\mu}+\sum_{j=1}^{d}\left(D_{j} \widetilde{v}, \tilde{a}_{j} \bar{\psi}_{j i}\right)_{\mu}=0, \quad i=1, \cdots, d .
$$

If we substitute in this equation $\widetilde{\chi}_{i}^{\beta}$ for $\widetilde{v}$, and then pass to the limit once along $\beta^{(1)}$ and once along $\beta^{(2)}$, and then subtract the results, we obtain:

$$
\sum_{x}\left(\bar{\xi}_{x, i}, \bar{\xi}_{x, i}\right)_{\mu}+\sum_{j=1}^{d}\left(\bar{\psi}_{j i}, \tilde{a}_{j} \bar{\psi}_{j i}\right)_{\mu}=0, \quad i=1, \cdots, d .
$$

Uniqueness, then follows from the above equation and (3.4).

We define

$$
q_{i j} \stackrel{\text { def. }}{=} \boldsymbol{E} \widetilde{a}_{i}\left(\delta_{i j}+\widetilde{\psi}_{i j}\right), \quad i, j=1, \cdots, d .
$$

Lemma 3.1. The "effective" diffusion matrix $q$ defined above is a positive definite symmetric matrix.

Proof. From Eq.(3.9), we have:

$$
\sum_{x}\left(\partial_{x} \widetilde{v}, \widetilde{\xi}_{x, i}\right)_{\mu}+\sum_{k=1}^{d}\left(D_{k} \widetilde{v}, \widetilde{a}_{k}\left(\delta_{k i}+\widetilde{\psi}_{k i}\right)\right)_{\mu}=0 .
$$

Substituting $\widetilde{v}=\widetilde{\chi}_{j}^{\beta}$ in this equation, and passing to the limit $\beta \downarrow 0$, we obtain

$$
\sum_{x}\left(\widetilde{\xi}_{x, j}, \widetilde{\xi}_{x, i}\right)_{\mu}+\sum_{k=1}^{d}\left(\widetilde{\psi}_{k j}, \widetilde{a}_{k}\left(\delta_{k i}+\widetilde{\psi}_{k i}\right)\right)_{\mu}=0
$$

Adding $q_{j i}=\sum_{k}\left(\delta_{k j}, \tilde{a}_{k}\left(\delta_{k i}+\widetilde{\psi}_{k i}\right)\right)_{\mu}$ to both sides,

$$
q_{j i}=\sum_{x \in \mathbb{Z}^{d}}\left(\widetilde{\xi}_{x, j}, \widetilde{\xi}_{x, i}\right)_{\mu}+\sum_{k=1}^{d}\left(\delta_{k j}+\widetilde{\psi}_{k j}, \widetilde{a}_{k}\left(\delta_{k i}+\widetilde{\psi}_{k i}\right)\right)_{\mu}
$$

This shows that $q$ is symmetric. Let $\mathbf{c}=\left(c_{1}, \ldots, c_{d}\right) \in \mathbb{R}^{d}$ be a nonzero arbitrary vector. Multiplying both sides of the above equation by $c_{i} c_{j}$ and summing over $i, j$, we obtain:

$$
\begin{aligned}
\sum_{i, j=1}^{d} c_{i} q_{i j} c_{j}=\sum_{x} & \left(\sum_{k=1}^{d} c_{k} \widetilde{\xi}_{x, k}, \sum_{k=1}^{d} c_{k} \widetilde{\xi}_{x, k}\right)_{\mu}+ \\
& \sum_{k=1}^{d}\left(\sum_{i=1}^{d}\left(\widetilde{\psi}_{k i}+\delta_{k i}\right) c_{i}, \tilde{a}_{k} \sum_{i=1}^{d}\left(\widetilde{\psi}_{k i}+\delta_{k i}\right) c_{i}\right)_{\mu}
\end{aligned}
$$


On the other hand,

$$
\boldsymbol{E}\left[\sum_{i=1}^{d}\left(\widetilde{\psi}_{k i}+\delta_{k i}\right) c_{i}\right]^{2}=\boldsymbol{E}\left(\sum_{i=1}^{d} \widetilde{\psi}_{k i} c_{i}\right)^{2}+\sum_{i=1}^{d} c_{i}^{2} \geq \sum_{i=1}^{d} c_{i}^{2}
$$

for $\boldsymbol{E}\left(\widetilde{\psi}_{k i}\right)=0$, which follows from the definition of $\widetilde{\psi}_{k i}=\lim _{\beta \downarrow 0} D_{k} \widetilde{\chi}_{i}^{\beta}$. Using the above inequality, (3.11) and (3.4),

$$
\sum_{i, j=1}^{d} c_{i} q_{i j} c_{j} \geq a_{0}^{-1} \sum_{i=1}^{d} c_{i}^{2}
$$

Similarly,

$$
\sum_{i, j=1}^{d} c_{i} q_{i j} c_{j} \leq\left(\sum_{i=1}^{d} c_{i}^{2}\right)\left\{\sum_{i=1}^{d} \sum_{x} \boldsymbol{E}\left(\widetilde{\xi}_{x, k}^{2}\right)+a_{0}\left(1+\sum_{j, k=1}^{d} \boldsymbol{E}\left(\widetilde{\psi}_{j k}\right)^{2}\right)\right\}
$$

So far we have constructed the effective diffusion matrix $q$ and we have shown that it is a positive definite matrix. The following estimates will play crucial roles:

\section{Proposition 3.1.}

(a) $\lim _{\beta \downarrow 0} \beta E\left(\widetilde{\chi}_{i}^{\beta}\right)^{2}=0, i=1, \cdots, d$,

(b) $\lim _{\beta \downarrow 0} E\left|D_{j} \widetilde{\chi}_{i}^{\beta}-\widetilde{\psi}_{j i}\right|^{2}=0$.

Proof. Subtracting Eq.(3.9) from Eq.(3.7), and substituting $\widetilde{v}=\widetilde{\chi}_{i}^{\beta}$ in the result, we obtain:

$$
\sum_{x}\left(\partial_{x} \tilde{\chi}_{i}^{\beta}, \partial_{x} \widetilde{\chi}_{i}^{\beta}-\widetilde{\xi}_{x, i}\right)_{\mu}+\sum_{j=1}^{d}\left(D_{j} \tilde{\chi}_{i}^{\beta}, \tilde{a}_{j}\left(D_{j} \tilde{\chi}_{i}^{\beta}-\widetilde{\psi}_{j i}\right)_{\mu}+\beta\left(\widetilde{\chi}_{i}^{\beta}, \tilde{\chi}_{i}^{\beta}\right)_{\mu}=0\right.
$$

Put $\widetilde{v}=\tilde{\chi}_{i}^{\beta}$ in Eq. (3.9) to obtain:

$$
\sum_{x}\left(\partial_{x} \widetilde{\chi}_{i}^{\beta}, \widetilde{\xi}_{x, i}\right)_{\mu}+\sum_{j=1}^{d}\left(D_{j} \widetilde{\chi}_{i}^{\beta}, \tilde{a}_{j} \tilde{\psi}_{j i}\right)_{\mu}=-\left(\tilde{a}_{i}, D_{i} \widetilde{\chi}_{i}^{\beta}\right)_{\mu}
$$

Passing to limit $\beta \downarrow 0$ :

$$
\sum_{x}\left(\widetilde{\xi}_{x, i}, \widetilde{\xi}_{x, i}\right)_{\mu}+\sum_{j=1}^{d}\left(\widetilde{\psi}_{j i}, \widetilde{a}_{j} \widetilde{\psi}_{j i}\right)_{\mu}=-\left(\widetilde{a}_{i}, \widetilde{\psi}_{i i}\right)_{\mu}
$$

Subtracting the last two equations yields

$$
\sum_{x}\left(\widetilde{\xi}_{x, i}, \partial_{x} \widetilde{\chi}_{i}^{\beta}-\widetilde{\xi}_{x, i}\right)_{\mu}+\sum_{j=1}^{d}\left(\widetilde{\psi}_{j i}, \widetilde{a}_{j}\left(D_{j} \widetilde{\chi}_{i}^{\beta}-\widetilde{\psi}_{j i}\right)\right)_{\mu}=-\left(\widetilde{a}_{i}, D_{i} \widetilde{\chi}_{i}^{\beta}-\widetilde{\psi}_{i i}\right)_{\mu}
$$

Finally subtract Eq.(3.13) from (3.12) to obtain: 


$$
\begin{array}{r}
\sum_{x}\left(\partial_{x} \widetilde{\chi}_{i}^{\beta}-\widetilde{\xi}_{x, i}, \partial_{x} \widetilde{\chi}_{i}^{\beta}-\widetilde{\xi}_{x, i}\right)_{\mu}+\sum_{j=1}^{d}\left(D_{j} \tilde{\chi}_{i}^{\beta}-\widetilde{\psi}_{j i}, \tilde{a}_{j}\left(D_{j} \widetilde{\chi}_{i}^{\beta}-\widetilde{\psi}_{j i}\right)\right)_{\mu}+ \\
\beta\left(\tilde{\chi}_{i}^{\beta}, \tilde{\chi}_{i}^{\beta}\right)_{\mu}=\left(\tilde{a}_{i}, D_{i} \tilde{\chi}_{i}^{\beta}-\widetilde{\psi}_{i i}\right)_{\mu} .
\end{array}
$$

Now pass to limit $\beta \downarrow$ 0. Proposition follows from (3.4), and the weak convergence of $\widetilde{\psi}_{j i}$.

Introduce the error

$$
z^{\varepsilon}(x, \varphi) \stackrel{\text { def. }}{=} u^{\varepsilon}(x, \varphi)-w^{\varepsilon}(x)+\varepsilon \sum_{i=1}^{d} \chi_{i}^{\varepsilon^{2}}(x / \varepsilon, \varphi) \nabla_{i}^{\varepsilon *} w^{\varepsilon}(x), \quad x \in \mathbb{Z}_{\varepsilon}^{d}
$$

where $\chi_{i}^{\beta}(x, \varphi) \stackrel{\text { def. }}{=} \tilde{\chi}_{i}^{\beta}\left(\tau_{-x} \varphi\right)$ satisfies:

$$
\left(v, \varepsilon\left(\mathscr{D}^{\varepsilon}+1\right) \chi_{i}^{\varepsilon^{2}}(x / \varepsilon, \varphi)\right)_{\varepsilon}=\left(v,-\nabla_{i}^{\varepsilon *} a_{i}(x / \varepsilon, \varphi)\right)_{\varepsilon}, \quad \forall v \in H_{\varepsilon}^{1}\left(\mathbb{Z}_{\varepsilon}^{d}, \mu\right) .
$$

We shall need the following result:

\section{Lemma 3.2.}

(a) $\left\|\varepsilon \chi_{i}^{\epsilon^{2}}(x / \varepsilon, \varphi) \nabla_{i}^{\varepsilon *} w^{\varepsilon}\right\|_{\varepsilon} \rightarrow 0$ as $\varepsilon \downarrow 0$.

Furthermore, there is a constant $C>0$, independent of $\varepsilon$, such that

(b) $\left\|z^{\varepsilon}\right\|_{\varepsilon} \leq C$,

(c) $\left\|\nabla_{i}^{\varepsilon} z^{\varepsilon}\right\|_{\varepsilon} \leq C$.

\section{Proof.}

(a)

$$
\begin{aligned}
\left\|\varepsilon \chi_{i}^{\varepsilon^{2}}(x / \varepsilon, \varphi) \nabla_{i}^{\varepsilon *} w^{\varepsilon}\right\|_{\varepsilon}^{2} & =\sum_{x}^{\varepsilon} \boldsymbol{E}\left(\varepsilon \chi_{i}^{\varepsilon^{2}}(x / \varepsilon, \varphi)\right)^{2}\left(\nabla_{i}^{\varepsilon *} w^{\varepsilon}\right)^{2} \\
& =\varepsilon^{2} \boldsymbol{E}\left(\widetilde{\chi}_{i}^{\varepsilon^{2}}\right)^{2} \sum_{x}^{\varepsilon}\left(\nabla_{i}^{\varepsilon *} w^{\varepsilon}\right)^{2} \rightarrow 0
\end{aligned}
$$

by Proposition 3.1.

(b)

$$
\left\|z^{\varepsilon}\right\|_{\varepsilon} \leq\left\|u^{\varepsilon}\right\|_{\varepsilon}+\|u\|_{\varepsilon}+\varepsilon \sum_{i=1}^{d}\left\|\chi_{i}^{\varepsilon^{2}}(x / \varepsilon, \varphi) \nabla_{i}^{\varepsilon *} w^{\varepsilon}\right\|_{\varepsilon} .
$$

The first term on the RHS is uniformly bounded in $\varepsilon$ by estimate (3.5). A similar estimate for $u$ bounds the second term and finally the last term is bounded uniformly by part (a).

(c) We have:

$$
\begin{array}{r}
\left\|\nabla_{i}^{\varepsilon} z^{\varepsilon}\right\|_{\varepsilon} \leq\left\|\nabla_{i}^{\varepsilon} u^{\varepsilon}\right\|_{\varepsilon}+\left\|\nabla_{i}^{\varepsilon} u\right\|_{\varepsilon}+\varepsilon \sum_{j=1}^{d}\left\|\left(\nabla_{i}^{\varepsilon} \chi_{j}^{\varepsilon^{2}}(x / \varepsilon, \varphi)\right) \nabla_{j}^{\varepsilon *} w^{\varepsilon}\left(x+\varepsilon \boldsymbol{e}_{i}\right)\right\|_{\varepsilon} \\
+\varepsilon \sum_{j=1}^{d}\left\|\chi_{j}^{\varepsilon^{2}}(x / \varepsilon, \varphi) \nabla_{i}^{\varepsilon} \nabla_{j}^{\varepsilon^{*}} w^{\varepsilon}(x)\right\|_{\varepsilon}
\end{array}
$$


The first two terms on the RHS are bounded, uniformly in $\varepsilon$. For the third term, we have:

$$
\left\|\varepsilon\left(\nabla_{i}^{\varepsilon} \chi_{j}^{\varepsilon^{2}}(x / \varepsilon, \varphi)\right) \nabla_{j}^{\varepsilon *} w^{\varepsilon}\left(x+\varepsilon e_{i}\right)\right\|_{\varepsilon}^{2}=E\left(D_{i} \tilde{\chi}_{j}^{\varepsilon^{2}}\right) \sum_{x}^{\varepsilon}\left(\nabla_{j}^{\varepsilon *} w^{\varepsilon}(x)\right)^{2},
$$

which is obviously bounded, uniformly in $\varepsilon$ (see (b) before Eq.(3.8)). The last term above is bounded by Proposition 3.1.

We are planning to calculate $\left(z^{\varepsilon},\left(\mathscr{D}^{\varepsilon}+\lambda\right) z^{\varepsilon}\right)_{\varepsilon}$. After some straightforward calculations, we obtain:

$$
\begin{aligned}
& \left(z^{\varepsilon},\left(\mathscr{S}^{\varepsilon}+\lambda\right) z^{\varepsilon}\right)_{\varepsilon}= \\
& \left(z^{\varepsilon}, \sum_{i, j=1}^{d}\left[q_{i j}-a_{j}^{\varepsilon} \delta_{i j}-\varepsilon a_{j}^{\varepsilon} \nabla_{j}^{\varepsilon} \chi_{i}^{\varepsilon^{2}}(x / \varepsilon, \varphi)\right] \nabla_{i}^{\varepsilon *} \nabla_{j}^{\varepsilon} w^{\varepsilon}\right)_{\varepsilon} \\
& +\left(z^{\varepsilon}, \sum_{i=1}^{d}\left[\varepsilon \mathscr{D}^{\varepsilon} \chi_{i}^{\varepsilon^{2}}(x / \varepsilon, \varphi)+\nabla_{i}^{\varepsilon *} a_{i}^{\varepsilon}(x)+\lambda \varepsilon \chi_{i}^{\varepsilon^{2}}(x / \varepsilon, \varphi)\right] \nabla_{i}^{\varepsilon *} w^{\varepsilon}\right)_{\varepsilon} \\
& +\sum_{i, j=1}^{d}\left(\nabla_{j}^{\varepsilon} z^{\varepsilon}, \varepsilon a_{j}^{\varepsilon} \chi_{i}^{\varepsilon^{2}}(x / \varepsilon, \varphi) \nabla_{j}^{\varepsilon} \nabla_{i}^{\varepsilon *} w^{\varepsilon}\right)_{\varepsilon} .
\end{aligned}
$$

That the last two terms vanish as $\varepsilon \downarrow 0$ follows easily from Eq. (3.15), Proposition 3.1 and Lemma 3.2. Therefore we only need to prove

Lemma 3.3. As $\varepsilon \downarrow 0$,

$$
I_{\varepsilon} \stackrel{\text { def. }}{=}\left(z^{\varepsilon}, \sum_{i, j=1}^{d}\left[q_{i j}-a_{j}^{\varepsilon} \delta_{i j}-\varepsilon a_{j}^{\varepsilon} \nabla_{j}^{\varepsilon} \chi_{i}^{\varepsilon^{2}}(x / \varepsilon, \varphi)\right] \nabla_{i}^{\varepsilon *} \nabla_{j}^{\varepsilon} w^{\varepsilon}\right)_{\varepsilon} \rightarrow 0 .
$$

Proof. Let $I_{\varepsilon}=I_{\varepsilon}^{\prime}+I_{\varepsilon}^{\prime \prime}$, where

$$
\begin{aligned}
& I_{\varepsilon}^{\prime} \stackrel{\text { def. }}{=}\left(z^{\varepsilon}, \sum_{i, j=1}^{d}\left[q_{i j}-a_{j}^{\varepsilon}\left(\delta_{i j}+\psi_{j i}(x / \varepsilon, \varphi)\right)\right] \nabla_{i}^{\varepsilon *} \nabla_{j}^{\varepsilon} w^{\varepsilon}\right)_{\varepsilon}, \\
& I_{\varepsilon}^{\prime \prime} \stackrel{\text { def. }}{=}\left(z^{\varepsilon}, \sum_{i, j=1}^{d} a_{j}^{\varepsilon}\left[\psi_{j i}(x / \varepsilon, \varphi)-\varepsilon a_{j}^{\varepsilon} \nabla_{j}^{\varepsilon} \chi_{i}^{\varepsilon^{2}}(x / \varepsilon, \varphi)\right] \nabla_{i}^{\varepsilon *} \nabla_{j}^{\varepsilon} w^{\varepsilon}\right)_{\varepsilon} .
\end{aligned}
$$

We shall show that $I_{\varepsilon}^{\prime}, I_{\varepsilon}^{\prime \prime} \rightarrow 0 . I_{\varepsilon}^{\prime \prime} \rightarrow 0$, as $\varepsilon \downarrow 0$ since

$$
\boldsymbol{E}\left\{\psi_{j i}(x / \varepsilon, \varphi)-\varepsilon \nabla_{j}^{\varepsilon} \chi_{i}^{\varepsilon^{2}}(x / \varepsilon, \varphi)\right\}^{2}=\boldsymbol{E}\left\{\tilde{\psi}_{j i}-D_{j} \tilde{\chi}_{i}^{\varepsilon^{2}}\right\}^{2} \rightarrow 0
$$

by Proposition 3.1. It remains to show that $I_{\varepsilon}^{\prime} \rightarrow 0$. Define

$$
\widetilde{g}_{i j}(\varphi) \stackrel{\text { def. }}{=} \widetilde{a}_{j}(\varphi)\left(q_{j i}-\delta_{j i}+\tilde{\psi}_{j i}(\varphi)\right)
$$


By definition, $E \tilde{g}_{j i}=0$, and we have

$$
I_{\varepsilon}^{\prime}=\left(z^{\varepsilon}, \sum_{i, j=1}^{d} g_{j i}(x / \varepsilon, \varphi) \nabla_{i}^{\varepsilon *} \nabla_{j}^{\varepsilon} w^{\varepsilon}\right)_{\varepsilon}
$$

where $g_{j i}(x, \varphi)=\widetilde{g}_{j i}\left(\tau_{-x} \varphi\right)$. Define

$$
G_{j i}^{k}(x, \varphi) \stackrel{\text { def. }}{=} \int \frac{\left(\mathrm{e}^{\mathrm{i} \lambda x}-1\right)\left(\mathrm{e}^{-\mathrm{i} \lambda_{k}}-1\right)}{\sum_{i}\left|\mathrm{e}^{\mathrm{i} \lambda_{i}}-1\right|^{2}} U(d \lambda) \widetilde{g}_{j i}(\varphi),
$$

where $\{U(d \lambda)\}_{\lambda \in \mathbb{R}^{d}}$ is the family of spectral projections corresponding to the family of unitary operators $\left\{T_{x}\right\}_{x \in \mathbb{Z}^{d}}$ :

$$
T_{x}=\int_{\mathbb{R}^{d}} \mathrm{e}^{\mathrm{i} \lambda x} U(d \lambda) .
$$

$G$ is well defined since

$$
\frac{\left|\mathrm{e}^{\mathrm{i} \lambda x}-1\right|\left|\mathrm{e}^{-\mathrm{i} \lambda_{k}}-1\right|}{\sum_{i=1}^{d}\left|\mathrm{e}^{\mathrm{i} \lambda_{i}}-1\right|^{2}} \leq 4 \sum_{i=1}^{d}\left|x_{i}\right|
$$

and we have

$$
\sum_{i=1}^{d} \nabla_{k} G_{j i}^{k}(x, \varphi)=g_{j i}(x, \varphi) .
$$

Moreover, since $U\left(d \lambda^{\prime}\right) U(\lambda)=U(\lambda)$ for $\lambda \in d \lambda^{\prime},=0$ otherwise, we have $\left(x \in \mathbb{Z}_{\varepsilon}^{d}\right)$ :

$$
\varepsilon^{2} \boldsymbol{E}\left(G_{j i}^{k}(x / \varepsilon, \varphi)\right)^{2} \leq \int_{\mathbb{R}^{d}} \varepsilon^{2} \frac{\left|\mathrm{e}^{\mathrm{i} \lambda x / \varepsilon}-1\right|^{2}}{\sum_{i}\left|\mathrm{e}^{\mathrm{i} \lambda_{i}}-1\right|^{2}}\left(U(d \lambda) \tilde{g}_{j i}, \tilde{g}_{j i}\right)_{\mu} .
$$

One can easily show that the integrand is bounded uniformly in $\varepsilon$ (for $x$ in any compact set):

$$
\varepsilon^{2} \frac{\left|\mathrm{e}^{\mathrm{i} \lambda x / \varepsilon}-1\right|^{2}}{\sum_{i}\left|\mathrm{e}^{\mathrm{i} \lambda_{i}}-1\right|^{2}} \leq 4 d^{2}|x|^{2}
$$

Since

$$
\lim _{\varepsilon \downarrow 0} \varepsilon^{2} \frac{\left|\mathrm{e}^{\mathrm{i} \lambda x / \varepsilon}-1\right|^{2}}{\sum_{i}\left|\mathrm{e}^{\mathrm{i} \lambda_{i}}-1\right|^{2}}=0, \quad \text { for } \lambda \notin 2 \pi \mathbb{Z}^{d},
$$

the dominated convergence theorem implies that

$$
\lim _{\varepsilon \downarrow 0} \varepsilon^{2} \boldsymbol{E}\left(G_{j i}^{k}(x / \varepsilon, \varphi)\right)^{2}=\lim _{\varepsilon \downarrow 0} \sum_{\lambda \in 2 \pi \mathbb{Z}^{d}} \varepsilon^{2} \frac{\left|\mathrm{e}^{\mathrm{i} \lambda x / \varepsilon}-1\right|^{2}}{\sum_{i}\left|e^{\mathrm{i} \lambda_{i}}-1\right|^{2}}\left(U(d \lambda) \widetilde{g}_{j i}, \widetilde{g}_{j i}\right)_{\mu}
$$

On the other hand, for $\lambda \in 2 \pi \mathbb{Z}^{d}, U(\lambda) \widetilde{g}_{j i}$ is $T_{x}$-invariant:

$$
T_{x} U(\lambda) \widetilde{g}_{j i}=\int_{\mathbb{R}^{d}} \mathrm{e}^{\mathrm{i} \lambda^{\prime} x} U\left(d \lambda^{\prime}\right) U(\lambda) \widetilde{g}_{j i}=U(\lambda) \widetilde{g}_{j i},
$$

since $\mathrm{e}^{\mathrm{i} \lambda x}=1$. Ergodicity of $T_{x}$, then, implies that $U(d \lambda) \widetilde{g}_{j i}$ is a constant and we have 


$$
\left(U(d \lambda) \widetilde{\boldsymbol{g}}_{j i}, \widetilde{\boldsymbol{g}}_{j i}\right)_{\mu}=U(d \lambda) \widetilde{\boldsymbol{g}}_{j i} \boldsymbol{E}\left(\widetilde{g}_{j i}\right)=0
$$

Hence

$$
\lim _{\varepsilon \downarrow 0} \varepsilon^{2} E\left(G_{j i}^{k}(x / \varepsilon, \varphi)\right)^{2}=0 .
$$

Therefore

$$
\begin{aligned}
&\left|\left(z^{\varepsilon}, g_{j i}(x / \varepsilon, \varphi) \nabla_{i}^{\varepsilon *} \nabla_{j}^{\varepsilon} w^{\varepsilon}\right)_{\varepsilon}\right|=\left|\sum_{k=1}^{d}\left(z^{\varepsilon} \nabla_{i}^{\varepsilon *} \nabla_{j}^{\varepsilon} w^{\varepsilon}, \varepsilon \nabla_{k}^{\varepsilon} G_{j i}^{k}(x / \varepsilon, \varphi)\right)_{\varepsilon}\right| \\
&=\left|\sum_{k=1}^{d}\left(\nabla_{k}^{\varepsilon *}\left(z^{\varepsilon} \nabla_{i}^{\varepsilon *} \nabla_{j}^{\varepsilon} w^{\varepsilon}\right), \varepsilon G_{j i}^{k}(x / \varepsilon, \varphi)\right)_{\varepsilon}\right| \\
& \rightarrow 0,
\end{aligned}
$$

because of $\left\|\nabla_{k}^{\varepsilon *} z^{\epsilon}\right\|_{\varepsilon} \leq C$ and Eq.(3.17).

Proof (Theorem 3.1). We have shown that

$$
\lim _{\varepsilon \downarrow 0}\left(z^{\varepsilon},\left(\mathscr{D}^{\varepsilon}+\lambda\right) z^{\varepsilon}\right)_{\varepsilon}=0
$$

that is,

$$
\lambda \lim _{\varepsilon \downarrow 0}\left(z^{\varepsilon}, z^{\varepsilon}\right)_{\varepsilon}=0 .
$$

Therefore $\left\|z^{\varepsilon}\right\|_{\varepsilon} \rightarrow 0$ as $\varepsilon \downarrow 0$. On the other hand, we have already observed that

$$
\left\|\varepsilon \chi_{i}^{\varepsilon^{2}}(x / \varepsilon, \varphi) \nabla_{i}^{\varepsilon *} w^{\varepsilon}\right\|_{\varepsilon} \rightarrow 0,
$$

by Proposition 3.1. These together yield $\left\|u^{\varepsilon}-w^{\varepsilon}\right\|_{\varepsilon} \rightarrow 0$.

3.2. Step 2: Removing mass. Theorem 3.1 proves the homogenization for the massive case, i.e. when $\lambda>0$. Since we will need to consider the case $\lambda=0$, let us, for $\lambda>0$, call the solutions to Eqs. (3.3) and (3.6), $u_{\lambda}^{\varepsilon}$ and $u_{\lambda}$, respectively.

Now consider the following PDE's:

$$
\begin{aligned}
\frac{-1}{\varepsilon^{2}} \Delta_{\varphi} u_{0}^{\varepsilon}+\sum_{i=1}^{d} \nabla_{i}^{\varepsilon *} a_{i}^{\varepsilon} \nabla_{i} u_{0}^{\varepsilon} & =\nabla_{\ell}^{\varepsilon *} f \\
-\sum_{i, j=1}^{d} q_{i j} \frac{\partial^{2} u_{0}}{\partial x_{i} \partial x_{j}} & =-\frac{\partial f}{\partial x_{\ell}} .
\end{aligned}
$$

In Sect. 2.1, we saw that Eq.(3.18) had a unique solution in the space $\mathfrak{D}_{\varepsilon}^{1}\left(\mathbb{Z}_{\varepsilon}^{d}, \mu\right)$. The second PDE (3.19) can be solved in the space $\mathscr{D}\left(\mathbb{R}^{n}\right)$, the completion of $C_{0}^{\infty}\left(\mathbb{R}^{d}\right)$ with respect to the norm

$$
\|u\|_{\mathscr{D}}^{2} \stackrel{\text { def. }}{=} \sum_{i=1}^{d} \int\left|\frac{\partial u(x)}{\partial x_{i}}\right|^{2} d x .
$$

Again the Lax-Milgram theorem shows the existence and uniqueness of the solution in this space. 
Next we will show that

Theorem 3.2. For any function $g \in C_{0}^{\infty}\left(\mathbb{R}^{n}\right)$ and any $\ell^{\prime}, 1 \leq \ell^{\prime} \leq d$, we have:

$$
\lim _{\varepsilon \downarrow 0} E\left\{\left(\sum_{x}^{\varepsilon}\left[u_{0}^{\varepsilon}(x, \varphi)-u_{0}(x)\right] \nabla_{\ell^{\prime}}^{\varepsilon *} g(x)\right)^{2}\right\}=0 .
$$

Proof. We break the above expression into three parts and write:

$$
\lim _{\lambda \downarrow 0} \lim _{\varepsilon \downarrow 0} E\left\{\left(I_{\varepsilon, \lambda}^{1}+I_{\varepsilon, \lambda}^{2}+I_{\varepsilon, \lambda}^{3}\right)^{2}\right\}
$$

where

$$
\begin{aligned}
I_{\varepsilon, \lambda}^{1} & =\sum_{x}^{\varepsilon}\left[u_{0}^{\varepsilon}(x, \varphi)-u_{\lambda}^{\varepsilon}(x, \varphi)\right] \nabla_{\ell^{\prime}}^{\varepsilon *} g(x), \\
I_{\varepsilon, \lambda}^{2} & =\sum_{x}^{\varepsilon}\left[u_{\lambda}^{\varepsilon}(x, \varphi)-u_{\lambda}(x)\right] \nabla_{\ell^{\prime}}^{\varepsilon *} g(x), \\
I_{\varepsilon, \lambda}^{3} & =\sum_{x}^{\varepsilon}\left[u_{\lambda}(x)-u_{0}(x)\right] \nabla_{\ell^{\prime}}^{\varepsilon *} g(x) .
\end{aligned}
$$

From Theorem 3.1, it follows that

$$
\lim _{\lambda \downarrow 0} \lim _{\varepsilon \downarrow 0} E\left(I_{\varepsilon, \lambda}^{2}\right)=0 .
$$

Therefore we are left with two more terms. We show that uniformly in $\varepsilon$,

(a) $\lim _{\lambda \downarrow 0}\left\langle\left(I_{\varepsilon, \lambda}^{1}(x, \varphi)\right)^{2}\right\rangle=0$,

(b) $\lim _{\lambda \downarrow 0}\left\langle\left(I_{\varepsilon, \lambda}^{3}(x)\right)^{2}\right\rangle=0$.

For this, we treat $d>2$ and $d \leq 2$ separately.

$(d>2)$ In this case, (a) follows from the uniform upper bound that was obtained in Sect. 2.2.2

$$
\begin{aligned}
\left|I_{\varepsilon, \lambda}^{1}(x, \varphi)\right| & \leq\left|\sum_{x}^{\varepsilon}\left[u_{0}^{\varepsilon}(x, \varphi)-u_{\lambda}^{\varepsilon}(x, \varphi)\right] \nabla_{\ell^{\prime}}^{\varepsilon *} g(x)\right| \\
& =\mid \sum_{x, y}^{\varepsilon} \int d \mu\left(\varphi^{\prime}\right) \int_{0}^{\infty} d t \mathrm{e}^{t / \varepsilon^{2} \Delta_{\varphi}-t \nabla^{\varepsilon *} a^{\varepsilon} \nabla^{\varepsilon}}\left(x, y ; \varphi, \varphi^{\prime}\right) \times \\
& \left(1-\mathrm{e}^{-t \lambda}\right) \nabla_{\ell}^{\varepsilon *} f(x) \nabla_{\ell^{\prime}}^{\varepsilon *} g(y) \mid \\
& \leq C \iiint d x d y d t \frac{\mathrm{e}^{-\|x-y\|^{2} / C t}}{t^{d / 2}}\left(1-\mathrm{e}^{-t \lambda}\right)\left|\frac{\partial f(x)}{\partial x_{\ell}} \frac{\partial g(y)}{\partial y_{\ell^{\prime}}}\right|
\end{aligned}
$$

where $C=C\left(a_{0}, d\right)$ can be chosen independent of $\varepsilon$, and we have used the fact that $\exp \left\{t \Delta_{\varphi}\right\} 1=1$. Since $f, g \in C_{0}^{\infty}$, the above integral converges to zero, as $\lambda \downarrow 0$, by dominated convergence theorem. This implies (a). Proof of (b) follows along the same lines. 
$(d=2)$ Without loss of generality, we can assume that $0<\theta \leq a_{i}(x, \varphi)<1$, for some constant $\theta$, uniformly in $x, \varphi$. This can be achieved by straightforward algebraic means. Let

$$
\begin{gathered}
K_{\lambda}^{\varepsilon} \stackrel{\text { def. }}{=} \nabla^{\varepsilon}\left(\mathscr{L}^{\varepsilon}+\lambda\right)^{-1} \nabla^{\varepsilon *}, \\
K_{\lambda}^{\varepsilon, 0} \stackrel{\text { def. }}{=} \nabla^{\varepsilon}\left(-1 / \varepsilon^{2} \Delta_{\varphi}-\Delta^{\varepsilon}+\lambda\right)^{-1} \nabla^{\varepsilon *},
\end{gathered}
$$

where $-\Delta^{\varepsilon}$ is the discrete Laplacian on the lattice $\mathbb{Z}_{\varepsilon}^{d}$. We will use the notation of Appendix A. Using the identity

$$
\begin{gathered}
K_{0}^{\varepsilon}-K_{\lambda}^{\varepsilon} \equiv\left(1+K_{0}^{\varepsilon} a^{\varepsilon}\right) A_{\lambda}^{\varepsilon}\left(1+a^{\varepsilon} K_{\lambda}^{\varepsilon}\right), \\
A_{\lambda}^{\varepsilon} \stackrel{\text { def. }}{=} K_{0}^{\varepsilon, 0}-K_{\lambda}^{\varepsilon, 0},
\end{gathered}
$$

we can rewrite

$$
I_{\varepsilon, \lambda}^{1}=\sum_{x}^{\varepsilon} g(x)\left[\left(1+K_{0}^{\varepsilon} a^{\varepsilon}\right) A_{\lambda}^{\varepsilon}\left(1+a^{\varepsilon} K_{l}^{\varepsilon} a\right) f\right](x, \varphi) .
$$

We know from Appendix A that both $\left(1+K_{0}^{\varepsilon} a^{\varepsilon}\right)$ and $\left(1+a^{\varepsilon} K_{\lambda}^{\varepsilon}\right)$ map $\ell^{s} \equiv$ $\ell^{s}\left(\mathbb{Z}_{\varepsilon}^{d}, L^{2}(\mu)\right)$ into itself, uniformly in $\varepsilon$ (i.e. their operator norms are bounded independent of $\varepsilon$ ), for $s$ close enough to 2 . If we view $A_{\lambda}^{\varepsilon}$ as an operator form $\ell^{p}$ to $\ell^{r}$, then we claim that its norm vanishes uniformly in $\varepsilon$, as $\lambda \downarrow 0$, for $p$ and $r$ less than (but close enough to) 2. Part (a), then, follows from this claim since

$$
\left|K_{0}^{\varepsilon}-K_{\lambda}^{\varepsilon}\right|_{p \rightarrow r} \leq\left|1+K_{0}^{\varepsilon} a^{\varepsilon}\right|_{r \rightarrow r} \cdot\left|A_{\lambda}^{\varepsilon}\right|_{p \rightarrow r} \cdot\left|1+a^{\varepsilon} K_{\lambda}^{\epsilon}\right|_{p \rightarrow p} \rightarrow 0,
$$

uniformly in $\varepsilon$ and this implies $I_{\varepsilon, \lambda}^{1} \rightarrow 0$ in $L^{2}(\mu)$. To prove our claim, we first note that $A_{\lambda}^{\varepsilon}$ acts as a convolution. For each fixed $x$, let $\bar{A}_{\lambda}^{\varepsilon}(x): L^{2}(\mu) \rightarrow L^{2}(\mu)$ be the kernel of $A_{\lambda}^{\varepsilon}$ and put $B_{\lambda}^{\varepsilon}(x)=\left\|\bar{A}_{\lambda}^{\varepsilon}(x)\right\|_{L^{2}(\mu) \rightarrow L^{2}(\mu)}$. From the generalized Young inequality for convolutions, we know that $\left|A_{\lambda}^{\varepsilon}\right|_{p \rightarrow r}$ is bounded by $\left\|B_{\lambda}^{\varepsilon}\right\|_{\ell^{\varepsilon}\left(\mathbb{Z}_{\varepsilon}^{2}\right)}$, where $p, q$ and $r$ are related through $1 / r=1 / p+1 / q-1$. So it suffices to prove that $\left\|B_{\lambda}^{\varepsilon}\right\|_{\ell q\left(Z_{\varepsilon}^{2}\right)}$ vanishes uniformly. This can be verified for $1<q<2$, if we note that $B_{\lambda}^{\varepsilon}$ in Fourier space looks like the $\lambda /\left(p^{2}+\lambda\right)$. Therefore

$$
\left|A_{\lambda}^{\varepsilon}\right|_{p \rightarrow r}=\left\|B_{\lambda}^{\varepsilon}\right\|_{\ell q\left(\mathbb{Z}_{\varepsilon}^{2}\right)} \rightarrow 0, \quad \text { as } \lambda \downarrow 0
$$

uniformly in $\varepsilon$, for $1<q<2$. We remark that we can not take $r=p$ since this implies $q=1$ and (3.20) is not true for $q=1$. However, a suitable choice of $q$, close enough to 1 , allows us to choose $r>p$, both close enough to 2 and this completes the proof of part (a).

Since convergence in $\lambda$ of these two terms is uniform in $\varepsilon$, we can replace the order of limits from $\lim _{\lambda \downarrow 0} \lim _{\varepsilon \downarrow 0}$ to $\lim _{\varepsilon \downarrow 0} \lim _{\lambda \downarrow 0}$ and then the theorem follows from the above observations. 


\section{Extensions to More General Interactions}

In this section, we extend the results of the previous section to the more general class of interactions with an arbitrary but finite range $R$. For this, we use a rather different notation which we now explain.

Let $\rho=\left(\rho_{1}, \ldots, \rho_{d}\right) \in \mathbb{Z}^{d}$. For a real (or complex) valued function $f(x)$ on $\mathbb{Z}_{\varepsilon}^{d}$, and $i=1, \ldots, d$, define

$$
\begin{gathered}
\left(\nabla_{\rho}^{\varepsilon} f\right)(x) \stackrel{\text { def. }}{=} \frac{f(x+\varepsilon \rho)-f(x)}{\varepsilon\|\rho\|}, \\
\left(\nabla_{\rho}^{\varepsilon *} f\right)(x) \stackrel{\text { def. }}{=} \frac{f(x-\varepsilon \rho)-f(x)}{\varepsilon\|\rho\|} .
\end{gathered}
$$

Note that $\nabla_{\rho}^{\varepsilon *}$ is the adjoint of $\nabla_{\rho}^{\varepsilon}$ with respect to the standard inner product of $\ell^{2}\left(\mathbb{Z}_{\varepsilon}^{d}\right)$. When $\varepsilon=1$, we shall drop the superscript $\varepsilon$. Put

$$
\mathbb{Z}_{+}^{d}(R) \stackrel{\text { def. }}{=}\left\{\left(x_{1}, \ldots, x_{d}\right) \in \mathbb{Z}^{d}:\|x\| \leq R, x_{i} \geq 0, i=1, \ldots, d\right\} .
$$

We will consider Hamiltonians of the type:

$$
H=\sum_{x} V\left(\left\{\|\rho\| \nabla_{\rho} \varphi(x): \rho \in \mathbb{Z}_{+}^{d}(R)\right\}\right),
$$

that is, for a fixed $x, V$ is a functional of all possible $\nabla_{\rho} \varphi(x)$ for all $\rho \in \mathbb{Z}_{+}^{d}(R)$. We assume that $V$ is at least a $C^{2}$ functional with respect to its arguments. Define

$$
\left.a_{\rho \sigma}(x, \varphi) \stackrel{\text { def. }}{=}\|\rho\| \cdot\|\sigma\| \frac{\partial^{2} V(z)}{\partial z_{\rho} \partial z_{\sigma}}\right|_{z=\left\{\left\|\rho^{\prime}\right\| \nabla_{\rho^{\prime}} \varphi(x)\right\}} .
$$

Our main conditions on the Hamiltonian will be

$$
\sum_{\rho \sigma} a_{\rho \sigma} c_{\rho} c_{\sigma} \geq \delta \sum_{i=1}^{d} c_{e_{i}}^{2}, \quad \text { for some constant } \delta>0
$$

for any vector $c=\left(c_{\rho}\right)_{\rho \in \mathbb{Z}_{+}^{d}(R)}$. We also require

$$
\left|a_{\rho \sigma}(x, \varphi)\right| \leq C,
$$

for some positive constant C, uniformly in $x$ and $\varphi$ (cf. (H-1) and (H-2)).

Any sum over small Greek letters is understood to run over the whole $\mathbb{Z}_{+}^{d}(R)$ unless stated otherwise.

Remark 10. Using B-L inequalities and (E-1), one can easily prove the existence of an infinite volume, translation invariant ergodic massless probability measure (cf. Sect. 1). 
Define

$$
\left\{\begin{array}{l}
\mathscr{L}^{\varepsilon} \stackrel{\text { def. }}{=} \frac{-1}{\varepsilon^{2}} \Delta_{\varphi}+\sum_{\rho \sigma} \nabla_{\rho}^{\varepsilon *} a_{\rho \sigma}^{\varepsilon} \nabla_{\sigma}^{\varepsilon *} \\
\widehat{\mathscr{D}}^{\varepsilon} \stackrel{\text { def. }}{=} \sum_{\rho \sigma} \widehat{q}_{\rho \sigma} \nabla_{\rho}^{\varepsilon *} \nabla_{\sigma}^{\varepsilon *} .
\end{array}\right.
$$

where $\widehat{q}_{\rho \sigma}$ will be defined shortly. Let

$$
D_{\rho} \stackrel{\text { def. }}{=} \frac{T_{\rho}-1}{\|\rho\|}, \quad D_{\rho}^{*} \stackrel{\text { def. }}{=} \frac{T_{-\rho}-1}{\|\rho\|} .
$$

For any function $g(x, \varphi)=T_{x} \widetilde{g}(\varphi)$, we have

$$
D_{\rho} g(x, \varphi)=\nabla_{\rho} g(x, \varphi), \quad D_{\rho}^{*} g(x, \varphi)=\nabla_{\rho}^{*} g(x, \varphi) .
$$

Then define $\widetilde{\chi}_{\sigma}^{\beta} \in \mathscr{H}^{1}(\mu), \sigma \in \mathbb{Z}_{+}^{d}(R)$ as the unique solution of

$$
\left(-\Delta_{\varphi}+\sum_{\rho \rho^{\prime}} D_{\rho}^{*} a_{\rho \rho^{\prime}} D_{\rho^{\prime}}+\beta\right) \chi_{\sigma}^{\beta}=-\sum_{\rho} D_{\rho}^{*} a_{\rho \sigma} .
$$

We shall work in the same Hilbert spaces as before. The Lax-Milgram theorem can be applied due to the (E-1) and (E-2). One obtains estimates similar to those following (3.7), which in turn, prove the existence of the weak limits

$$
\left\{\begin{array}{l}
\widetilde{\psi}_{\rho \sigma} \stackrel{\text { def. }}{=} \lim _{\beta \downarrow 0} D_{\rho} \widetilde{\chi}_{\sigma}^{\beta} \\
\widetilde{\xi}_{x \rho} \stackrel{\text { def. }}{=} \lim _{\beta \downarrow 0} \partial_{x} \widetilde{\chi}_{\sigma}^{\beta}
\end{array}\right.
$$

One can again show that these convergences take place in $L^{2}(\mu)$. We define

$$
\widehat{q}_{\rho \sigma}=E \sum_{\rho^{\prime}} a_{\rho \rho^{\prime}}\left(\delta_{\rho^{\prime} \sigma}+\widetilde{\psi}_{\rho^{\prime} \sigma}\right)
$$

and prove

Lemma 4.1. For $\widehat{q}$ defined above, we have

$$
\sum_{\sigma \sigma^{\prime}} q_{\sigma \sigma^{\prime}} c_{\sigma} c_{\sigma^{\prime}} \geq \delta \sum_{i} c_{e_{i}}^{2}
$$

Proof. As in the proof of Lemma 3.1, we will obtain

$$
\widehat{q}_{\sigma \sigma^{\prime}}=\sum_{x}\left(\widetilde{\xi}_{x \sigma}, \widetilde{\xi}_{x \sigma^{\prime}}\right)_{\mu}+\sum_{\rho \rho^{\prime}}\left(\widetilde{\psi}_{\rho \sigma}+\delta_{\rho \sigma}, a_{\rho \rho^{\prime}}\left(\widetilde{\psi}_{\rho^{\prime} \sigma^{\prime}}+\delta_{\rho^{\prime} \sigma^{\prime}}\right)\right)_{\mu}
$$

Multiplying by $c_{\sigma} c_{\sigma^{\prime}}$ and summing over indices, we obtain

$$
\sum_{\sigma \sigma^{\prime}} \widehat{q}_{\sigma \sigma^{\prime}} c_{\sigma} c_{\sigma^{\prime}}=\sum_{\rho \rho^{\prime}}\left(A_{\rho}, a_{\rho \rho^{\prime}} A_{\rho^{\prime}}\right)_{\mu}
$$

where $A_{\rho}=\sum_{\sigma} c_{\sigma}\left(\delta_{\rho \sigma}+\widetilde{\psi}_{\rho \sigma}\right)$. Hypothesis (E-1) then gives the lower bound

$$
\delta \sum_{i=1}^{d} A_{e_{i}}^{2}
$$

On the other hand, since $\boldsymbol{E} \widetilde{\psi}_{\rho \sigma}=0$, this last expression dominates $\delta \sum_{i} c_{\boldsymbol{e}_{i}}^{2}$. 
It is important to understand that this lemma implies the ellipticity of the limiting continuum operator. Let

$$
\frac{\partial}{\partial x} \stackrel{\text { def. }}{=}\left(\frac{\partial}{\partial x_{1}}, \ldots, \frac{\partial}{\partial x_{d}}\right)
$$

be the continuum gradient. Then as $\varepsilon \downarrow 0$, we have

$$
\nabla_{\rho}^{\varepsilon} \rightarrow \widehat{\rho} \cdot \frac{\partial}{\partial x}, \quad \widehat{\rho}=\frac{\rho}{\|\rho\|}
$$

hence

$$
\widehat{\mathscr{D}^{\varepsilon}} \rightarrow-\sum_{i j} q_{i j} \frac{\partial^{2}}{\partial x_{i} \partial x_{j}}
$$

where

$$
q_{i j} \stackrel{\text { def. }}{=} \sum_{\rho \rho^{\prime}} \widehat{q}_{\rho \rho^{\prime}} \widehat{\rho}_{i}{\widehat{\rho^{\prime}}}_{j}
$$

Ellipticity of $q$ is exactly equivalent to the above lemma. The error $z^{\varepsilon}$ now takes the form

$$
z^{\varepsilon}(x, \varphi) \stackrel{\text { def. }}{=} u^{\varepsilon}(x, \varphi)-w^{\varepsilon}(x, \varphi)+\varepsilon \sum_{\rho} \chi_{\rho}^{\varepsilon^{2}}(x / \varepsilon, \varphi) \nabla_{\rho}^{\varepsilon *} w^{\varepsilon}(x)
$$

where $u^{\varepsilon}$ and $w^{\varepsilon}$ satisfy

$$
\left\{\begin{array}{l}
\left(\mathscr{L}^{\varepsilon}+\lambda\right) u^{\varepsilon}(x, \varphi)=\nabla_{\ell}^{*} f(x) \\
\left(\sum_{\rho \sigma} q_{\rho \sigma} \nabla_{\rho}^{\varepsilon *} \nabla_{\sigma}^{\varepsilon}+\lambda\right) w^{\varepsilon}(x)=\nabla_{\ell}^{*} f(x)
\end{array}\right.
$$

The rest of proof goes through with obvious modifications and we will not repeat them here.

\section{A. $\ell^{p}$ Estimates}

In this appendix, we state some $\ell^{p}$ estimates on the solutions of the PDE's which were studied in previous sections. We start with some definitions and notations.

For a functional $u(x, \varphi)$, let us define its $\ell^{p}$ norm, $p>1$ by

$$
|u|_{p}^{p} \stackrel{\text { def. }}{=} \sum_{x}\|u(x, \cdot)\|^{p}
$$

where

$$
\|u(x, \cdot)\|^{2}=\left\langle|u(x, \varphi)|^{2}\right\rangle \equiv \int|u(x, \varphi)|^{2} d \mu(\varphi),
$$

and define $\ell^{p}\left(\mathbb{Z}^{2}, \mathscr{H}(\mu)\right)$ as the collection of functions $u$ with $|u|_{p}<\infty$. We denote the norm of an operator $A: \ell^{p}(\mathbb{Z}, \mathscr{F} B) \rightarrow \ell^{r}(\mathbb{Z}, \mathscr{F} B)$ by $|A|_{p \rightarrow r}$. Assume that $u \in$ $\ell^{1}\left(\mathbb{Z}^{d}, \mathscr{H}\right)$. Then we can define its Fourier transform

$$
\widehat{u}(y)=\sum_{x} \mathrm{e}^{\mathrm{i} p x} u(x),
$$

which is an element of $\ell^{\infty}\left(\mathbb{Z}^{d}, \mathscr{B}\right)$. If $u \in \ell^{1}\left(\mathbb{Z}^{d}, \mathscr{F}\right) \cap \ell^{2}\left(\mathbb{Z}^{d}, \mathscr{F}\right)$, then $\widehat{u} \in$ $\ell^{2}\left(\mathbb{Z}^{d}, \mathscr{F}\right)$, with $|u|_{2}=|\widehat{u}|_{2}$. 
Let $\mathscr{B}(\mathscr{H}(\mu))$ be the collection of all bounded linear maps on $\mathscr{H}(\mu)$ and denote the norm of an operator $T \in \mathscr{B}$ by $\|T\|$. Assume $u(x)$ takes values in $\mathscr{B}$ and $\mathscr{K}$ takes values in $\mathscr{B}(\mathscr{B}(\mu))$. Then

$$
T u(x) \stackrel{\text { def. }}{=} \sum_{y} \mathscr{K}(x-y) f(y),
$$

whenever defined, takes values in $\mathscr{H}(\mu)$. We have

Theorem . Let $\|\mathscr{B}(x)\| \in \ell^{2}\left(\mathbb{Z}^{d}\right)$. Suppose that

(a) The Fourier transform of $\mathscr{B}$ is essentially bounded

$$
\|\widehat{\mathscr{B}}\| \leq B \text {. }
$$

(b)

$$
\sum_{\|x\| \geq 2\|y\|}\|\mathscr{K}(x-y)-\mathscr{K}(x)\| \leq B, \quad\|y\|>0 .
$$

For $u \in L^{1}\left(\mathbb{Z}^{d}, \mathscr{H}\right) \cap \ell^{p}\left(\mathbb{Z}^{d}, \mathscr{H}\right)$, set

$$
T u(x)=\sum_{y} \mathscr{B}(x-y) f(y) .
$$

Then there exists a constant $A_{p}$, so that

$$
\|T u\|_{p} \leq A_{p}\|u\|_{p}, \quad 1<p<\infty .
$$

Therefore $T$ can be extended to all of $\ell^{p}$ by continuity. The constant $A_{p}$ depends only on $p, B$ and $d$.

For a proof, see Chapter 2 of [14]. Let, as before,

$$
\mathscr{B}=-\Delta_{\varphi}+\nabla^{*} a \nabla .
$$

We will assume that $0<\theta \leq a_{i}(x, \varphi)<1$. Therefore, we can write $\mathscr{L}=\mathscr{C}_{0}+\nabla^{*} b \nabla$, where

$$
\mathscr{L}_{0} \stackrel{\text { def. }}{=}-\Delta_{\varphi}-\Delta_{x}, \quad \text { and }\left|b_{i}\right| \leq 1-\theta .
$$

As a corollary of the above theorem, we have:

Corollary A.1. For any $p, 1<p<\infty, D^{0} \stackrel{\text { def. }}{=} \nabla \mathscr{B}_{0}^{-1} \nabla^{*}$ maps $\left(\ell^{p}\left(\mathbb{Z}^{d}, \mathscr{Z} \mathscr{b}\right)\right)^{d}$ into itself.

The following proposition shows that for $p$ close enough to 2 , the same conclusion is true for $\nabla \mathscr{L}^{-1} \nabla^{*}$.

Proposition A.1. Let $0<\theta \leq a_{i}(x, \varphi)<1, f \in C_{0}^{\infty}\left(\mathbb{R}^{d}\right)$. Then there exists a $p=p(\theta)>2$ such that $\nabla u \in \ell^{p}\left(\mathbb{Z}^{d}, \mathscr{B}\right)$, where $u$ satisfies

$$
\mathscr{B} u=\nabla_{\ell}^{*} f
$$

for any given compactly supported $f$. 
Proof. We can rewrite (A.1) as

$$
\sum_{j}\left(\delta_{i j}+D_{i j}^{0} b_{j}\right) \nabla_{j} u=D_{i \ell}^{0} f .
$$

Since the RHS is in $\ell^{p}\left(\mathbb{Z}^{d}, \mathscr{Z}\right)$, for any $p$, it suffices to show the existence of a $p>2$ such that

$$
\left\|D^{0} b\right\|_{\left(\ell^{p}\right)^{d}}<1 \text {. }
$$

Since $|b| \leq 1-\theta$, it suffices to show that $g(p) \stackrel{\text { def. }}{=}\left\|D^{0}\right\|_{\left(\ell^{p}\right)^{d}}$, which takes the value 1 at $p=2$, remains close enough to 1 as $p$ remains close to 2 . This can be easily seen using the Riesz-Thorin interpolation.

Acknowledgement. It is our pleasure to thank G. Papanicolaou, J. Sjöstrand and S.R.S. Varadhan for many helpful discussions. We also thank M. Avellaneda, H. Spohn and D. Stroock for their useful remarks.

\section{References}

1. Brascamp, H.J. and Lieb, E.: On extensions of the Brunn-Minkowski and Prekopa-Leinler theorems. J. Funct. Anal. 22, 366-389(1976)

2. Brydges, D. and Yau, H-T.: Grad $\varphi$ perturbations of massless Gaussian fields. Commun. Math. Phys. 129, 351-392(1990)

3. Carlen, E.A., Kusuoka, S. and Stroock, D.W.: Upper bounds for symmetric Markov transition functions. Ann. Inst. H. Poincaré. Probab. Statist., Sup. au no 2, 245-287(1987)

4. Gawedzki, K. and Kupiainen, A.: Renormalization group study of a critical lattice model, I and II. Commun. Math. Phys. 82, 407-433(1981)

5. Gilbarg, D. and Trudinger, N.S.: Elliptic Partial Differential Equations of Second Order. BerlinHeidelberg-New York: Springer Verlag, 1977

6. Guo, M. Z. and Papanicolaou, G.: Self-diffusion of interacting Brownian particles. Probabilistic methods in mathematical physics (Katata/Kyoto, 1985) Boston, MA: Academic Press, 1987, pp. 113-151

7. Helffer, B.: Spectral properties of the Kac operator in large dimensions. Preprint, LMENS-93-17, 1993

8. Helffer, B. and Sjöstrand, J.: On the correlation for Kac-like models in the convex case. J. Statist. Phys. 74(1/2), 349-409(1994)

9. Künnemann, R.: The diffusion limit for reversible jump processes on $\mathbb{Z}^{d}$ with ergodic random bond conductivities. Commun. Math. Phys. 90, 27-68(1983)

10. Magnen, J. and Sénéor, R.: The infrared behavior of $(\nabla \varphi)_{3}^{4}$. Ann. Physics 152, 136-202(1984)

11. Newman, C.: Normal fluctuations and the FKG inequalities. Commun. Math. Phys. 74, 119-128(1980)

12. Sjöstrand, J.: Correlation asymptotics and Witten Laplacians. To appear.

13. Funaki, T. and Spohn, H.: Motion by mean curvature from Ginzburg-Landau $\nabla \phi$ interface model. Preprint

14. Stein, Elias M.: Singular Integrals and Differentiability Properties of Functions. Princeton, NJ: Princeton University Press, 1970

15. Papanicolaou, G.C. and Varadhan, S.R.S.: Boundary value problems with rapidly oscillating random coefficients. Volume 2 of Coll. Math. Soc. Janos Bolya, 27. Random fields, Amsterdam, North Holland Publ. Co., 1981, pp. 835-873

Communicated by D.C. Brydges

This article was processed by the author using the $\mathrm{LAT}_{\mathrm{E}} \mathrm{X}$ style file pljourl from Springer-Verlag. 